\title{
Histochemistry of primate extraocular muscles and the changes of denervation
}

\author{
J. H. J. DURSTON \\ Institute of Neurology, London
}

The morphological and physiological classification of the fibres of the extraocular muscles has been the subject of some controversy, not to say confusion. It is well known that in mammals the oblique and rectus muscles consist of a peripheral layer of smaller fibres overlying a deeper zone with larger diameters (Kato, I938; Kern, I965). Many morphological and ultrastructural studies have described a dual type system in several species, including man, but later reports have suggested that this is an oversimplification. The application of histochemical methods to skeletal muscle has clarified the typing of the fibres and has enabled certain physiological properties to be inferred from their relative staining intensities. The purpose of the present study was to examine systematically control and denervated extraocular muscles in the baboon and control human material with a variety of histochemical and morphological techniques and to attempt to correlate the findings with those of other workers.

\section{Materials and methods}

Adult female olive baboons (Papio anubis) were used for control and experimental studies.

In nine animals, the right oculomotor nerve was sectioned in the middle cranial fossa through a temporal craniotomy under halothane anaesthesia. Local orbital trauma was thus avoided. Two baboons were killed after intervals of 3 weeks, 3 months, 6 months, and I year. One animal was killed 2 years after operation.

In two animals, the right oculomotor nerve was crushed for 10 minutes with watchmakers' forceps. These baboons were killed at 3 and 9 months respectively. The muscles of both orbits were removed under pentobarbitone anaesthesia just before death by overdose. Those of the left orbit were used as controls and the right superior oblique and lateral rectus muscles were also examined.

Control observations were also made on muscles from patients who had undergone orbital exenterations for conditions such as optic gliomata in which the muscles or their nerves had not been affected.

Paraffin blocks were made from the medial rectus muscles of the baboons and sections were stained with haematoxylin and eosin after fixation in Io per cent. formol saline. Lengths measuring $\mathrm{I}$ cm. from all the other muscles were embedded in gum tragacanth, frozen in isopentane cooled in liquid nitrogen, and sectioned at 8-10 $\mu$ in a Pearse-Slee cryostat (Engel and Cunningham, 1963).

The following staining techniques were used:

For morphological detail:

Haematoxylin and eosin

Modified Gomori trichrome (Engel and Cunningham, 1963).

For fibre typing :

Myofibrillar ATPase (Padykula and Herman, 1955) 
Myofibrillar ATPase with pre-incubation in sequestrate buffer at $\mathrm{pH} 4.35$ and haematoxylin counterstain (after Drews and Engel, i 966)

Myofibrillar ATPase with pre-incubation in veronal buffer at $\mathrm{pH}_{4} \cdot 6$ (Guth and Samaha, 1969)

Menadione-linked $\alpha$-glycerophosphate dehydrogenase (Wattenberg and Leong, 1960).

To illustrate the glycolytic pathway:

Periodic acid-Schiff for glycogen with haematoxylin counterstain

Phosphorylase (Takeuchi and Kuriaki, 1955).

To illustrate the oxidative pathway:

$\mathrm{NADH}_{2}$ tetrazolium reductase (Novikoff, Shin, and Drucker, I96I)

Succinate dehydrogenase (Nachlas, Tsou, de Souza, Cheng, and Seligman, 1957).

For neutral lipids:

Sudan black B.

For neuro-muscular junctions:

Non-specific esterases (Davis and Ornstein, 1959).

For capillaries:

Alkaline phosphatase (Romanul, r965).

Control and experimental muscles were subjected to identical procedures and processed in the same media. Identical areas of muscle were photographed from adjacent serial sections. All illustrations were taken from baboon material unless stated otherwise.

\section{Results}

(I) GONTROL MUSGLES

(a) Morphological observations

In the present study three distinct types of fibre were found in the extraocular muscles of the baboon and man. It is proposed to call them "coarse", "fine", and "granular" fibres according to their respective reticular patterns. The coarse fibres correspond to the Felderstruktur fibres of earlier studies, while the fine and the granular types represent two types of Fibrillenstruktur fibre. The main morphological features of the three fibre types are illustrated in Figs I and 2.

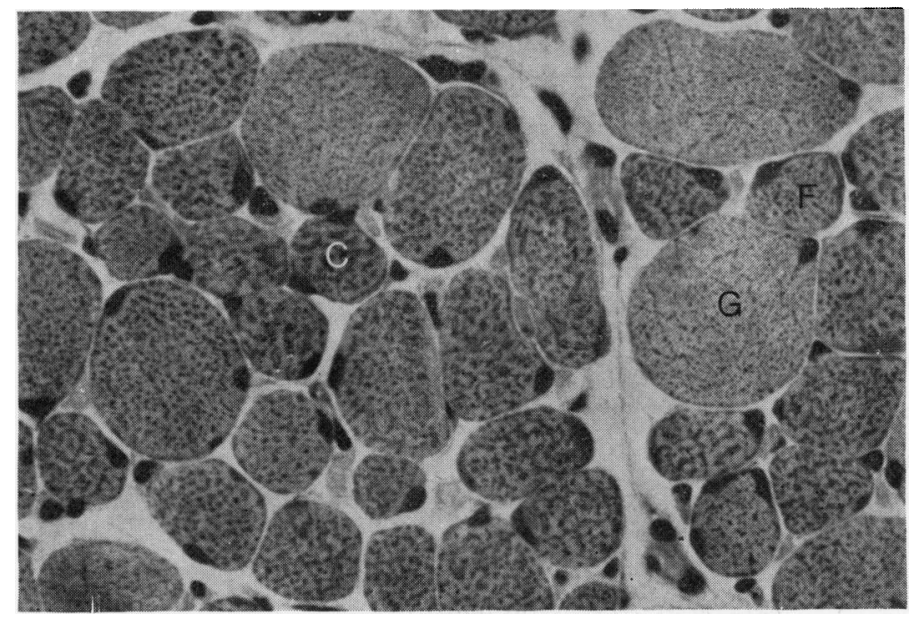

FIG. I Control levator palpebrae superioris. Haematoxylin and eosin. $\quad \times 34^{\circ}$

$\mathrm{C}=$ coarse fibre

$F=$ fine fibre

$\mathrm{G}=$ granular fibre 


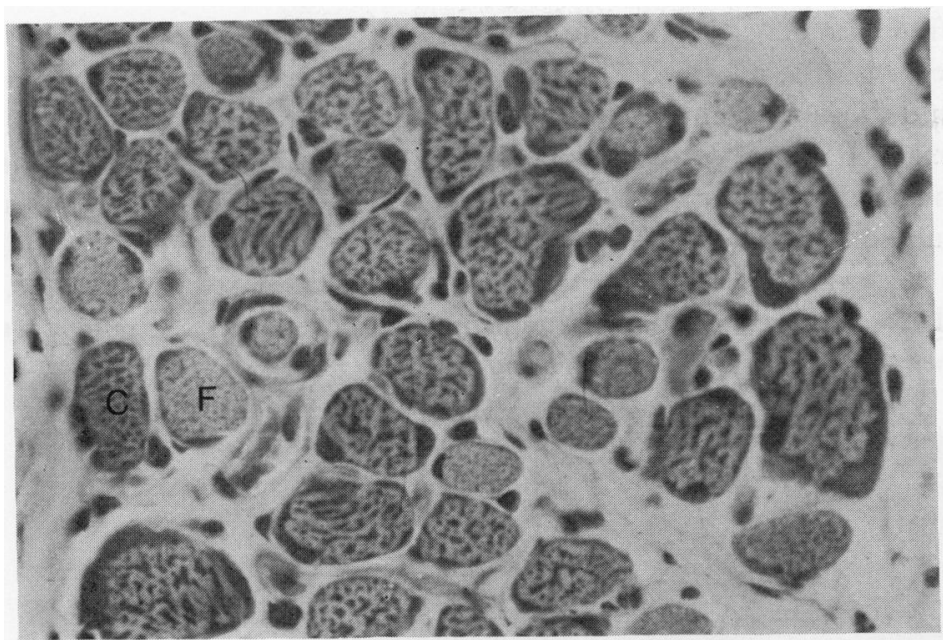

(a)

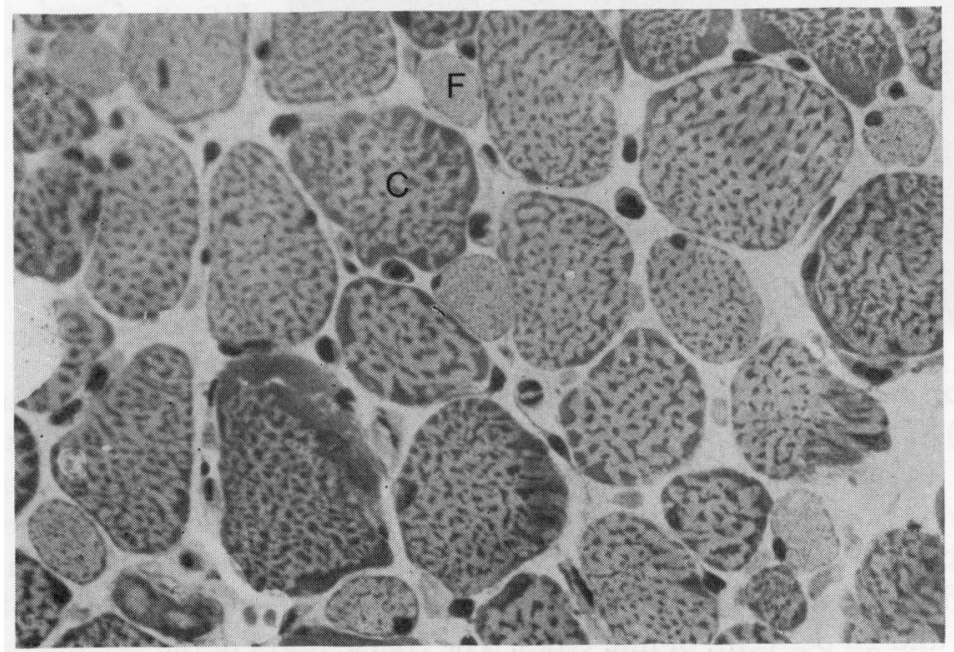

(b)

FIG. 2 Control inferior rectus. Haematoxylin and eosin. $\times 34^{\circ}$ $\mathbf{C}=$ coarse fibre $F=$ fine fibre $\mathbf{G}=$ granular fibre

(a) Peripheral zone

(b) Intermediate zone

(c) Central zone

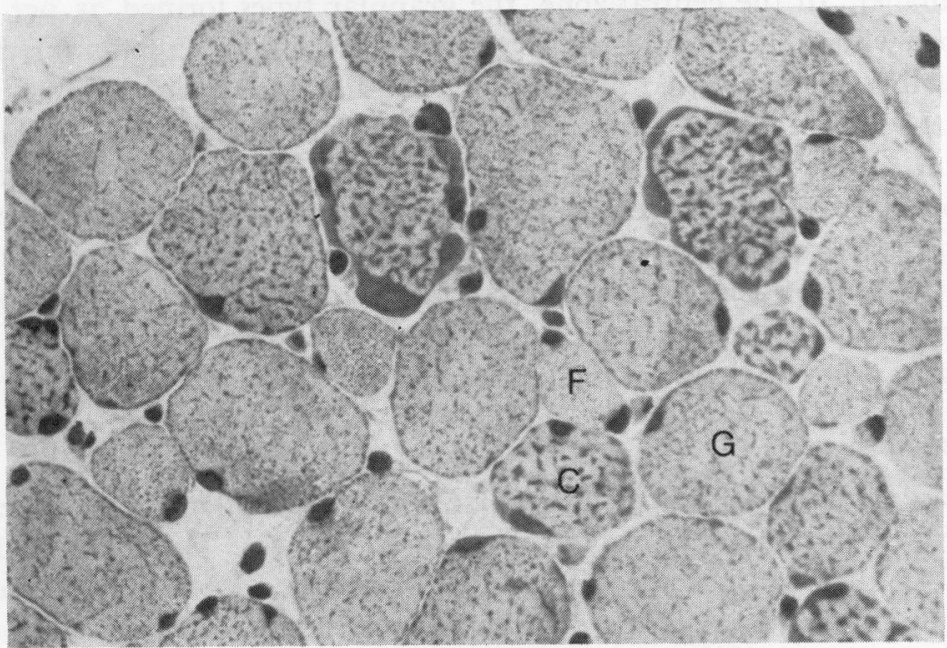

(c) 
The coarse fibres possessed a reticulum which was conspicuous, irregular, and bright red when stained with the modified trichrome technique. Sarcoplasmic masses were found in the central and subsarcolemmal regions. From one to three subsarcolemmal nuclei were seen in transverse section and occasional nuclei were centrally situated. The fine fibres were smaller and contained a regular, more delicate reticulum with one to three subsarcolemmal nuclei. They stained light green with trichrome and were markedly eosinophilic in frozen sections. In the larger granular fibres, the reticulum was delicate but irregular. These fibre types contained two or three subsarcolemmal nuclei. Degenerative changes were found in the reticulum of many fibres, particularly the coarse types (Fig. 3).

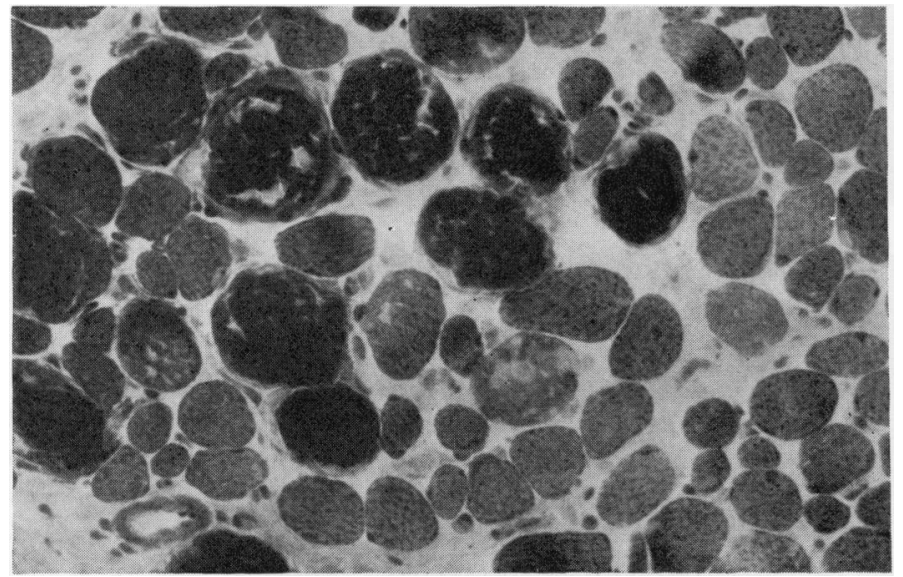

FIG. 3 Control inferior rectus. Modified Gomori trichrome. $\times$ 240. Degenerative changes

In the levator palpebrae superioris muscles, the three types were arranged in a random fashion (Fig. I). The coarse types formed 75 to 80 per cent. of the fibre population and the granular fibres 15 per cent. In the majority of these muscles, the fine fibres accounted for up to 5 per cent., but in two baboon muscles none was found. In the oblique and rectus muscles, the fibres were arranged in three zones, but the boundaries between them were not sharply defined (Fig. 2). The peripheral and intermediate zones contained 8o per cent. of coarse fibres and 20 per cent. of fine types. The coarse fibres of the peripheral zone were considerably smaller. In the central zone, the granular types formed 75 per cent. of the fibre population, while the fine and the coarse fibres accounted equally for the rest. The coarse fibres of this region showed a wide range of diameters, while the fine fibres were slightly larger than those of the outer zones. Large intramuscular nerves were seen in the central zones. Near the origins of these muscles, the peripheral and intermediate zones formed a double ring around the central zone when observed in transverse section. As the rectus and inferior oblique muscles were traced anteriorly, the ring configuration gave way to an arc and the central zone became directly apposed to the globe. These appearances were more obvious in low-power views of the histochemical preparations (Figs I4 and I5). The ring configuration remained throughout the length of the superior oblique muscles.

\section{(b) Histochemical observations}

With the ATPase reaction at $\mathrm{pH} \mathrm{9.4,} \mathrm{the} \mathrm{coarse} \mathrm{and} \mathrm{the} \mathrm{granular} \mathrm{fibres} \mathrm{stained} \mathrm{intensely,}$ while the fine fibres showed a relatively poor reaction (Fig. 4a). The coarse and the granular fibres could be distinguished, because in the former the myofibrils were separated by irregular sarcoplasmic material. These staining properties were reversed when the sections 


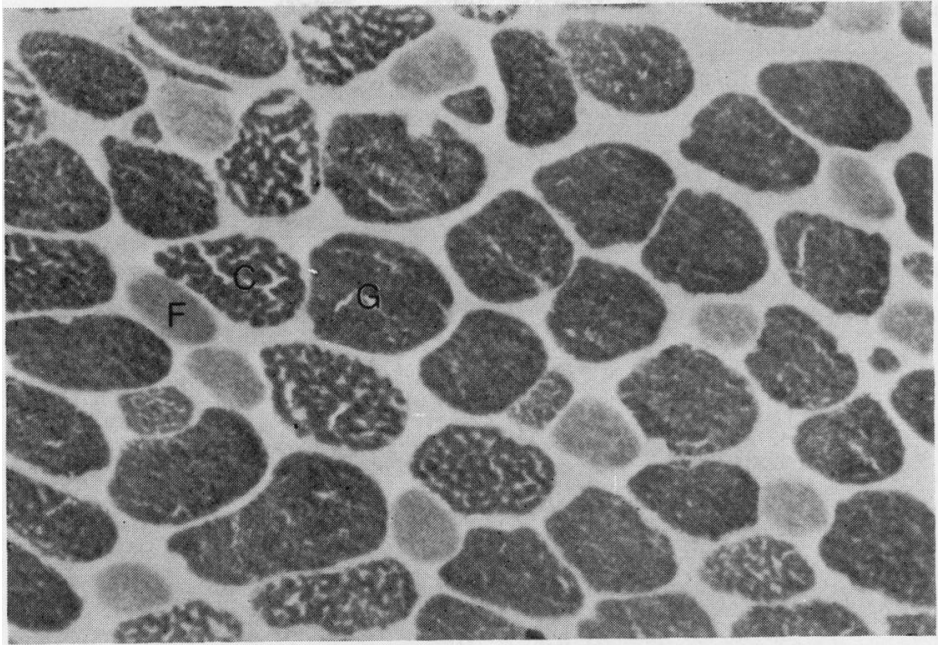

(a)

FIG. 4 Control superior rectus. Identical areas. Central zone. $\times 340$ $\mathrm{C}=$ coarse fibre $\mathbf{F}=$ fine fibre $\mathbf{G}=$ granular fibre

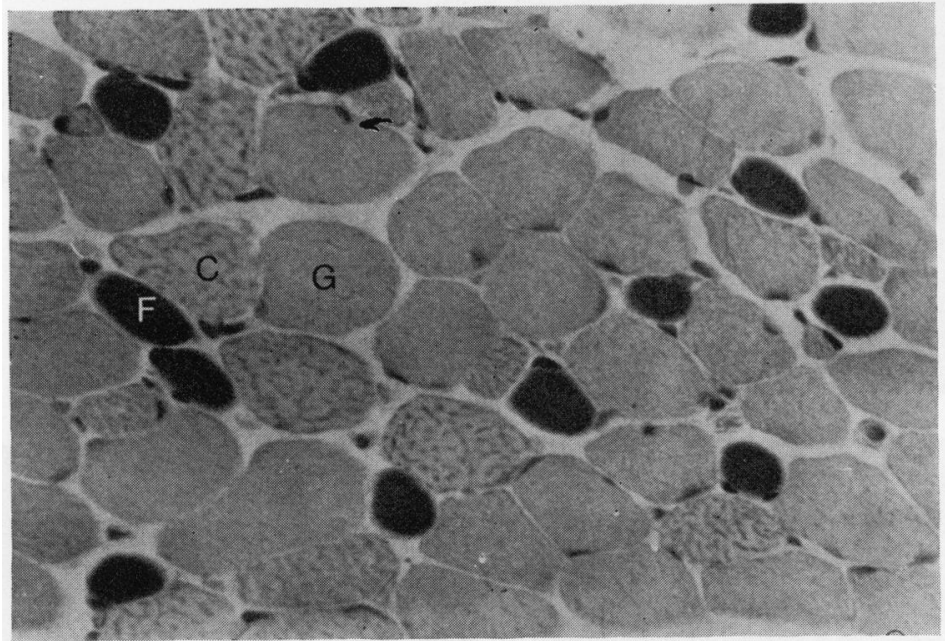

(a) ATPase at $\mathrm{pH} 9 \cdot 4$

(b) Acid-activated

ATPase (sequestrate, pH 4.35). Haematoxylin counterstain

were pre-incubated in acid buffer (Fig. $4 b$ ). The intensity for menadione-linked $\alpha-$ glycerophosphate dehydrogenase, a mitochondrial stain, was high in the granular fibres but low in the fine and the coarse types (Fig. $5^{b}$ ).

The $\mathrm{NADH}_{2}$ tetrazolium reductase and succinate dehydrogenase reactions confirmed the reticular patterns seen in the morphological preparations (Figs $5 c$ and $6 c$ ). The coarse fibres stained intensely, particularly in the peripheral zones of the oblique and rectus muscles, while the fine and the granular types showed a moderate to poor reaction. The neutral lipid content of the coarse fibres was also high. The granular fibres reacted least. The lipid content of the fine fibres of the obliques and recti was also low, but in the levator muscles the reaction was stronger (Figs $5^{d}$ and $6 b$ ).

When stained for glycogen and phosphorylase, the fine and the granular fibres showed a poor reaction in all zones and in all muscles. In the levator muscles, the coarse fibres showed a moderate reaction (Fig. $6 d$ ). In the oblique and rectus muscles, this fibre type reacted strongly in the peripheral zones but the staining intensities became progressively weaker towards the central regions (Fig. 7, overleaf).

The fine fibres showed the most intense reaction for non-specific esterases. The coarse 
(a)

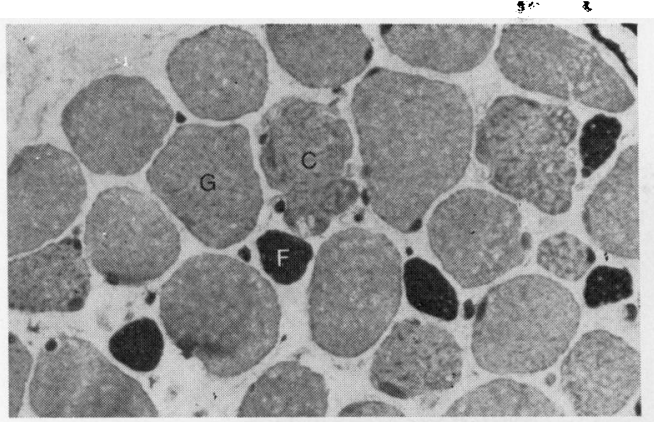

(c)

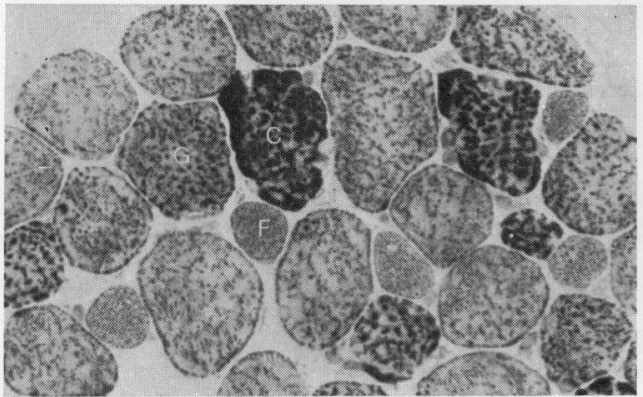

(e)

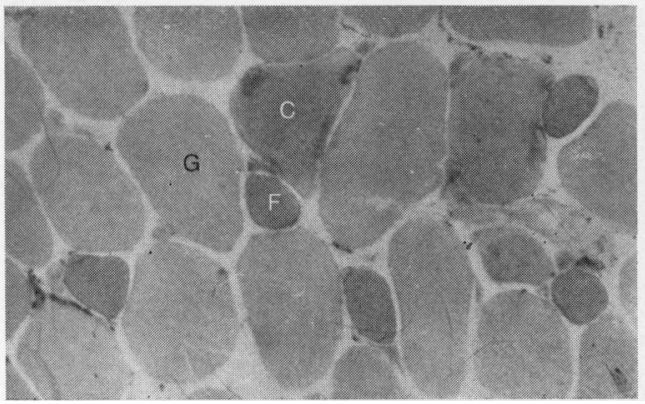

(a)

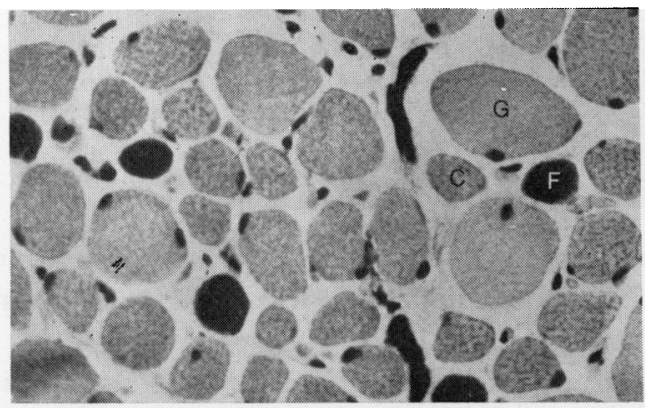

(c)

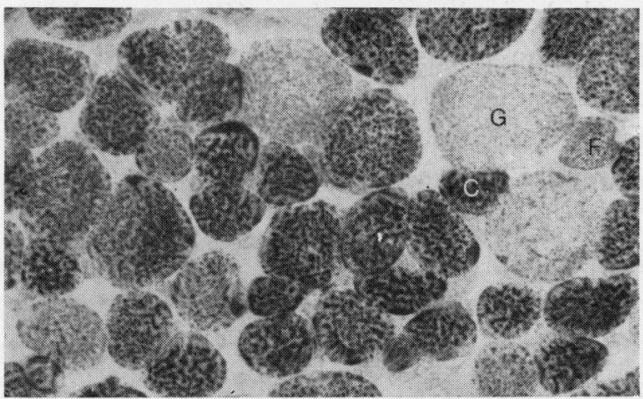

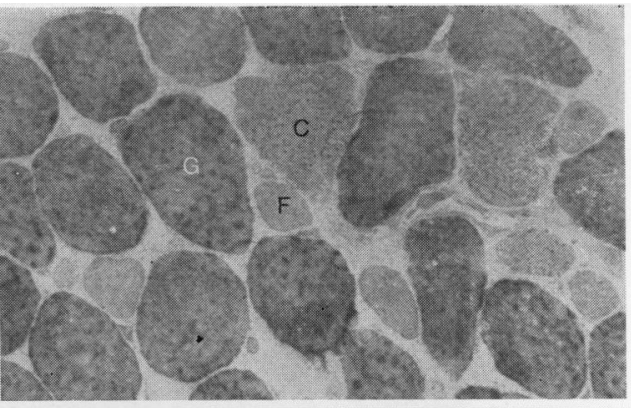

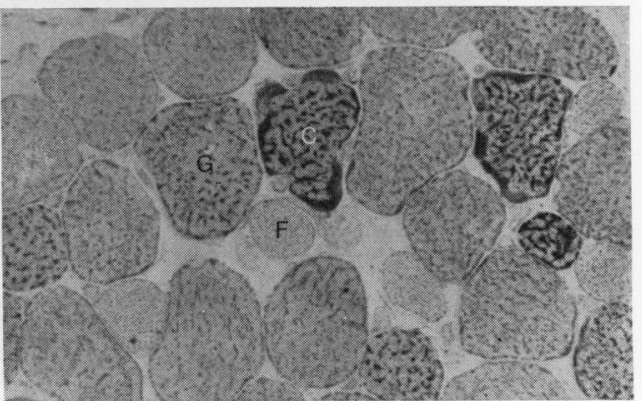

FI G. 5 Control inferior rectus. Identical areas. Central zone. $\quad \times 226$

(a) Acid-activated ATPase (sequestrate, $\mathrm{pH}$ 4.35). Haematoxylin counterstain

(b) Menadione-linked $\alpha$-glycerophosphate dehydrogenase

(c) $\mathrm{NADH}_{2}$ tetrazolium reductase

(d) Sudan black B

(e) Non-specific esterases
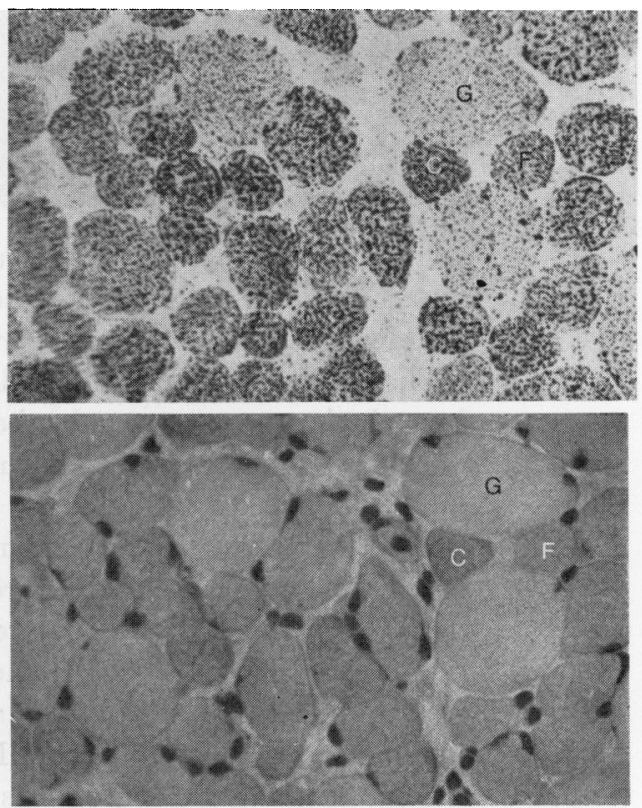

FIG. 6 Control levator palprebrae superioris. Identical areas. 226

(a) Acid-activated ATPase (sequestrate, $\mathrm{pH} 4 \cdot 35$ ). Haematoxylin counterstain

(b) Sudan black B

(c) Succinate dehydrogenase

(d) Periodic acid-Schiff. Haematoxylin counterstain 
(a)

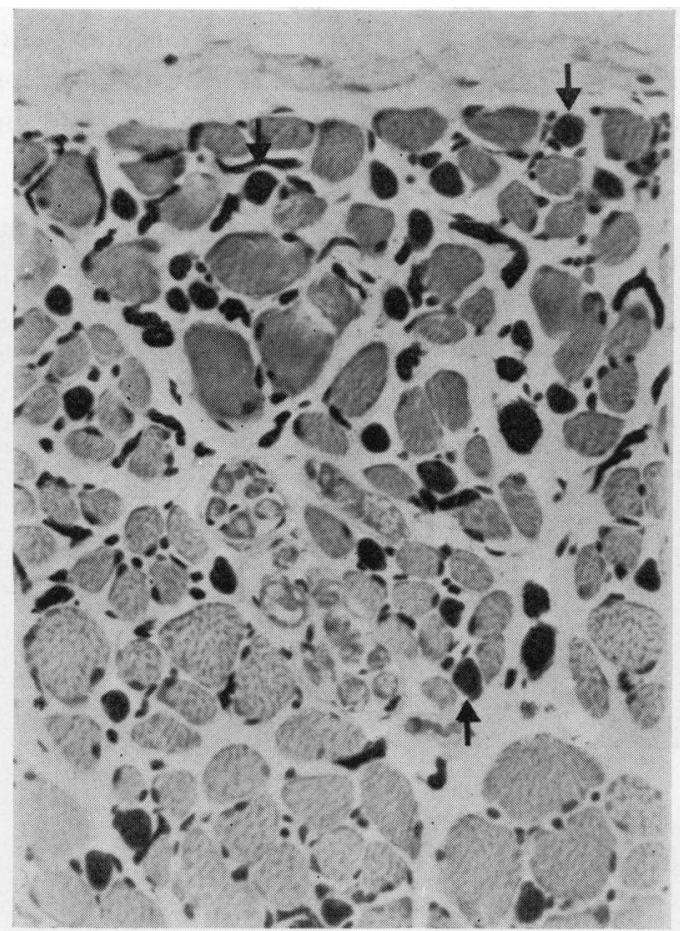

(c)

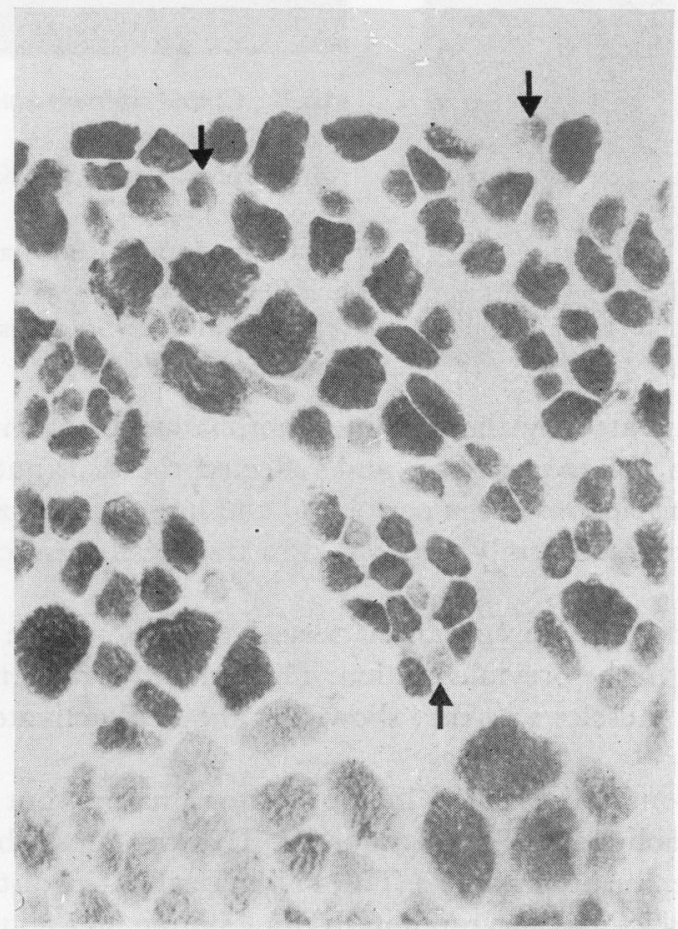

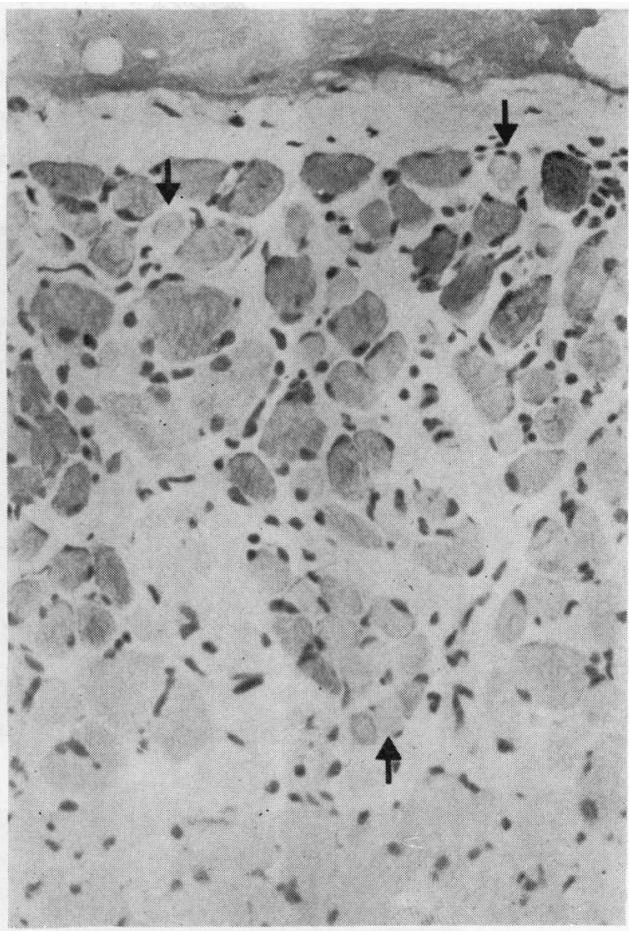

(b)
FI G. 7 Control superior rectus. Identical areas. Peripheral and intermediate zones. $\times 240$

(a) Acid-activated ATPase (sequestrate, $\mathrm{pH}$ 4.35). Haematoxylin counterstain. Fine fibres dark (arrowed), coarse fibres light

(b) Periodic acid-Schiff. Haematoxylin counterstain. Fine fibres stain poorly (arrowed). Coarse fibres stain intensely in peripheral zone and less strongly in intermediate zone

(c) Phosphorylase. Fine fibres stain poorly (arrowed). Coarse fibres stain intensely in peripheral zone and less strongly in intermediate zone 
fibres stained moderately, while the granular types showed little or no reaction (Fig. $5^{e}$ ). The esterase reaction also demonstrated the sites of the neuro-muscular junctions. The coarse fibres showed large loci of reaction product suggestive of multiple endings en grappe (Fig. 8a). Single endings en plaque were seen in the fine fibres (Fig. 8b). Endings en plaque were also found in the granular fibres but they were smaller (Fig. 8c).

(a)

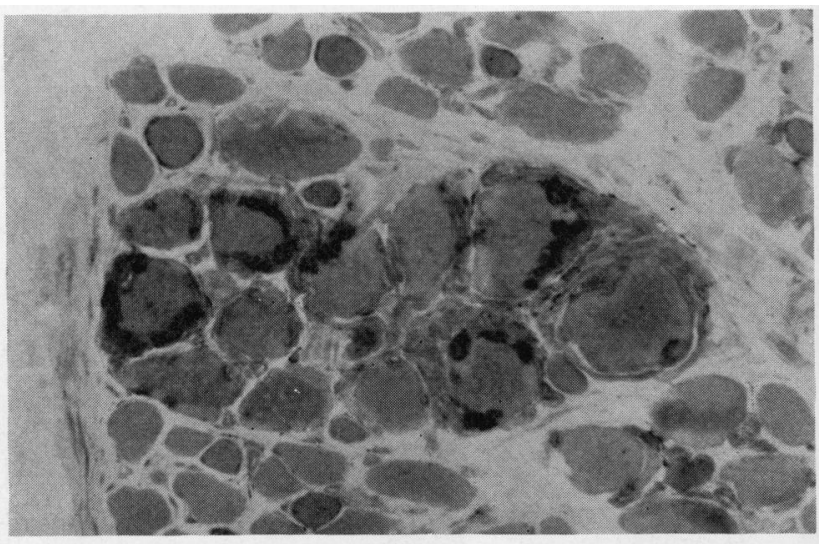

(b)
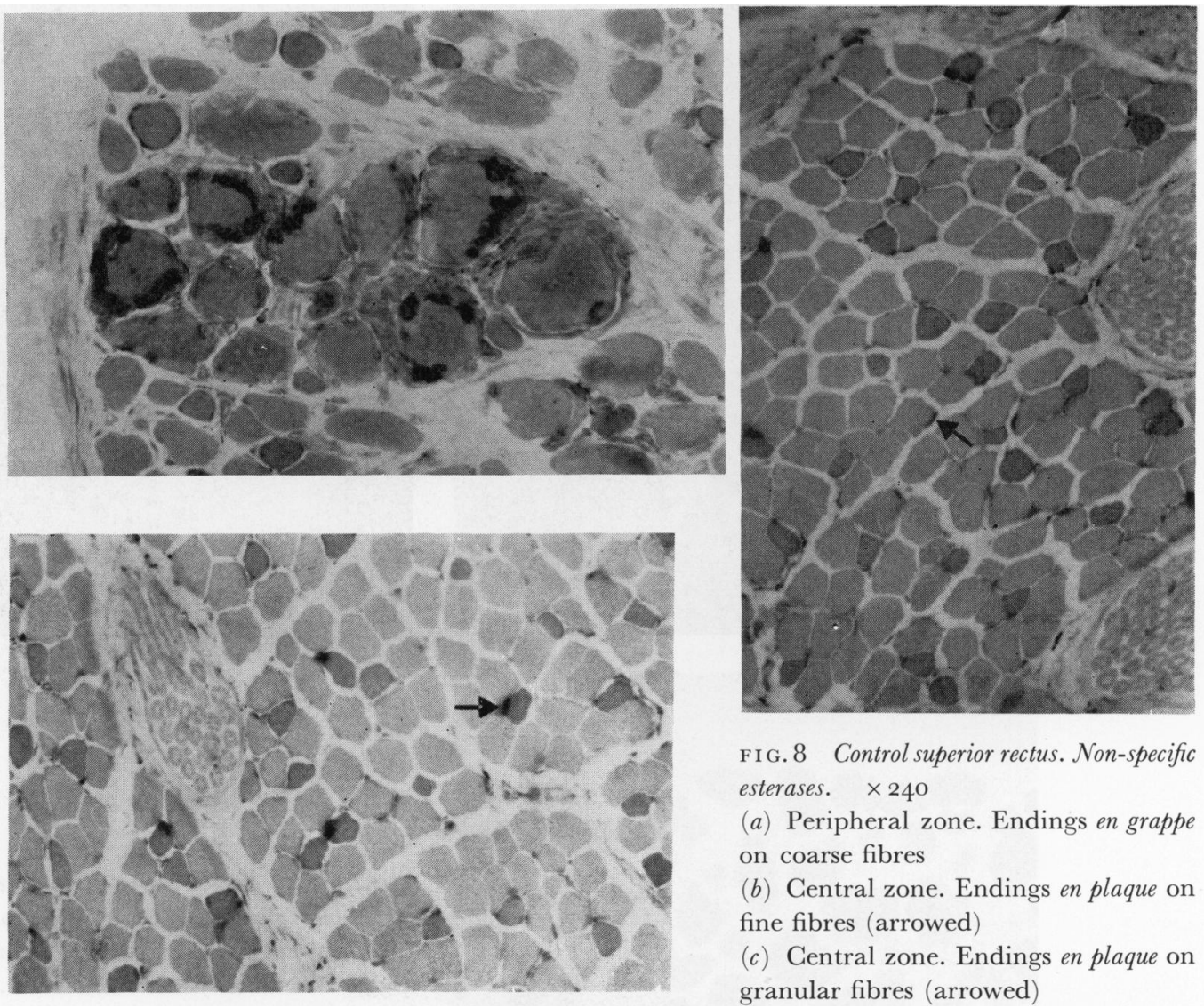

The capillary networks demonstrated by the alkaline phosphatase reaction were particularly prominent in relation to the coarse fibres and reflected the distribution of this fibre type. They were therefore conspicuous in the peripheral and intermediate zones of the oblique and rectus muscles, but were uniformly distributed in the levator muscles (Fig. 9, opposite).

Low-power views of the histochemical preparations served to demonstrate the distribution of the fibre types described in the previous section. The random arrangement of the fine fibres seen in all the control muscles was best shown by the acid-activated ATPase reaction (Fig. Io, opposite).

The granular fibres were randomly distributed in the levator muscles, but were confined to the central zones in the oblique and rectus muscles. This was most obvious with the $\alpha$-glycerophosphate dehydrogenase preparations (Fig. I I, overleaf). The distribution of the coarse fibres in the levator muscles was also random. In the oblique and rectus muscles they were found mostly in the peripheral and intermediate zones. These appearances were easily seen in the sections stained for lipids and oxidative activities (Fig. 12, overleaf). 
(a)
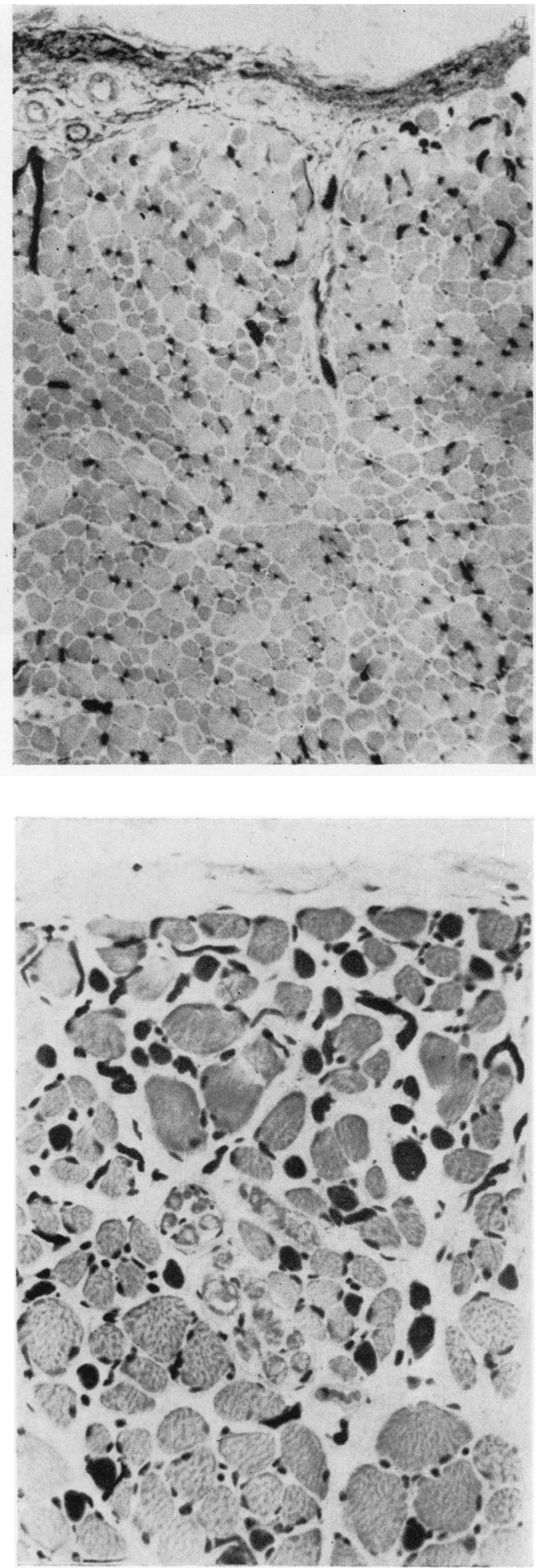

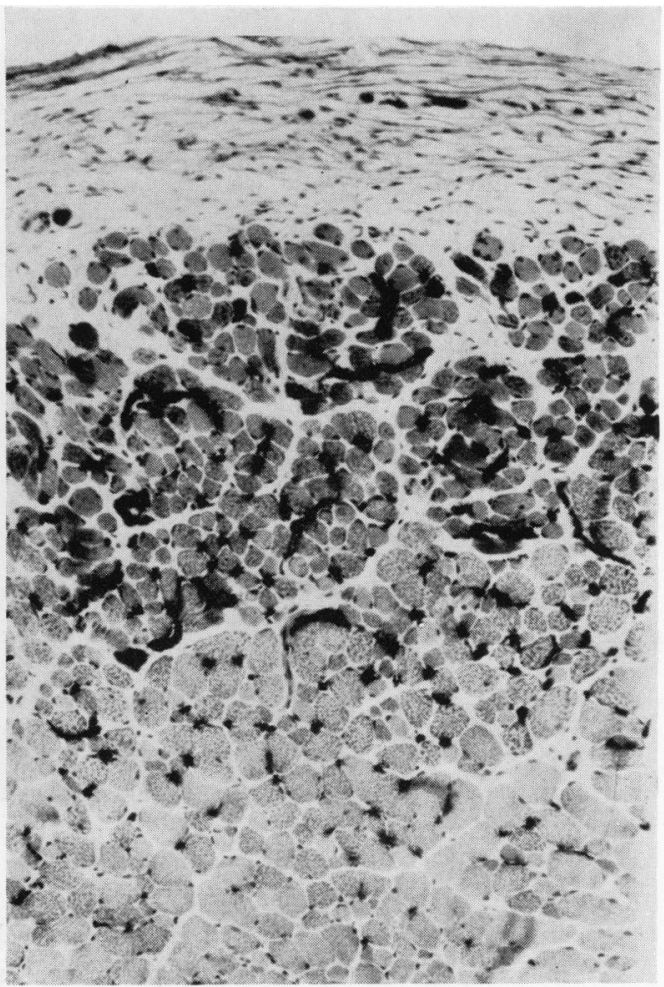

FIG. 9 Alkaline phosphatase. Distribution of capillaries. $\quad \times 80$

(a) Control levator palpebrae superioris

(b) Control superior rectus

FIG. I o Control superior rectus. Acid-activated ATPase (sequestrate, pH 4.35). Haematoxylin counterstain. $\times 80$. Distribution of fine fibres 

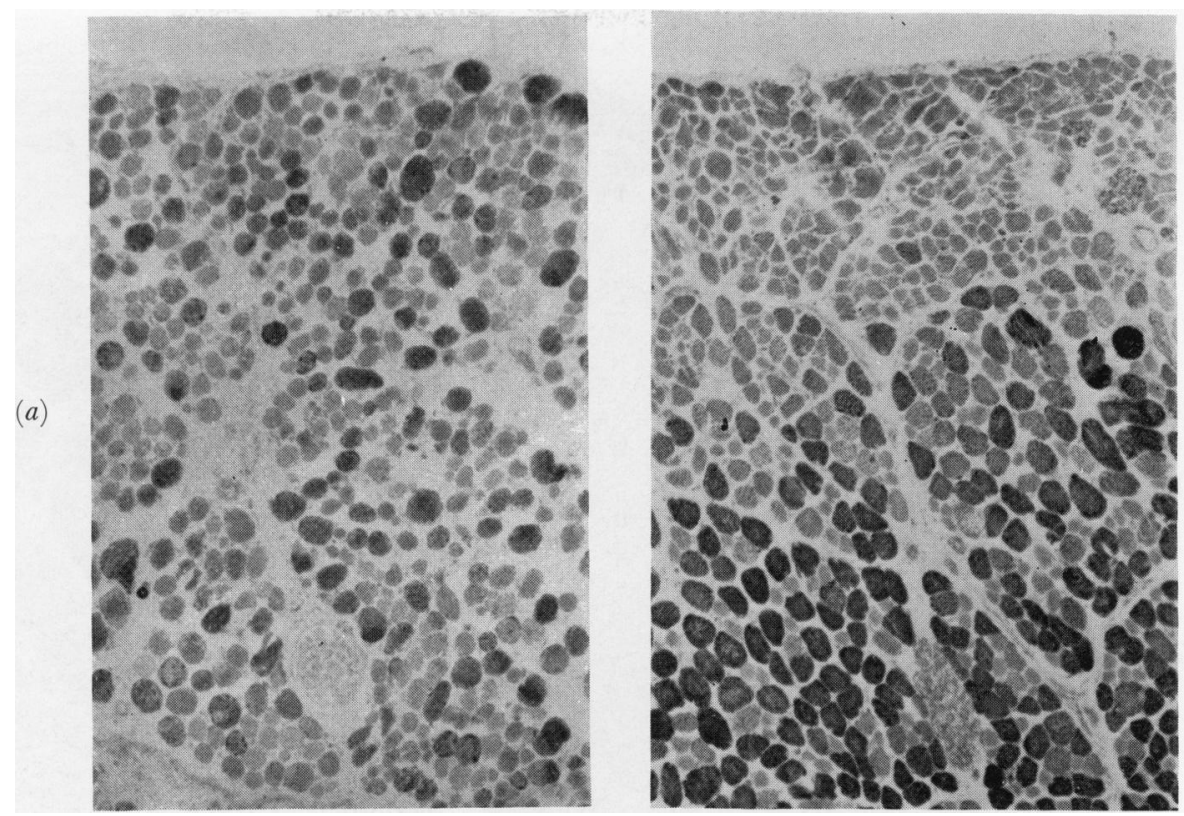

(b)

FIG. I I Menadione-linked $\alpha$-glycerophosphate dehydrogenase. $\times 80$. Distribution of granular fibres

(a) Control levator palprebrae superioris

(b) Control superior rectus

(a)

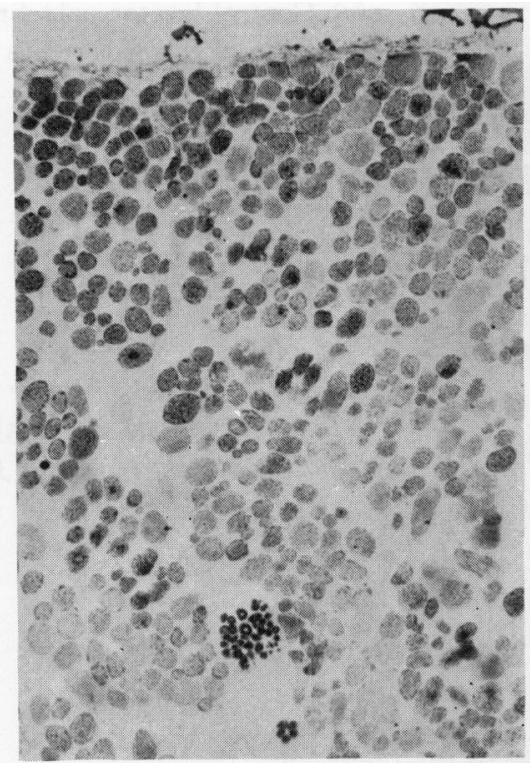

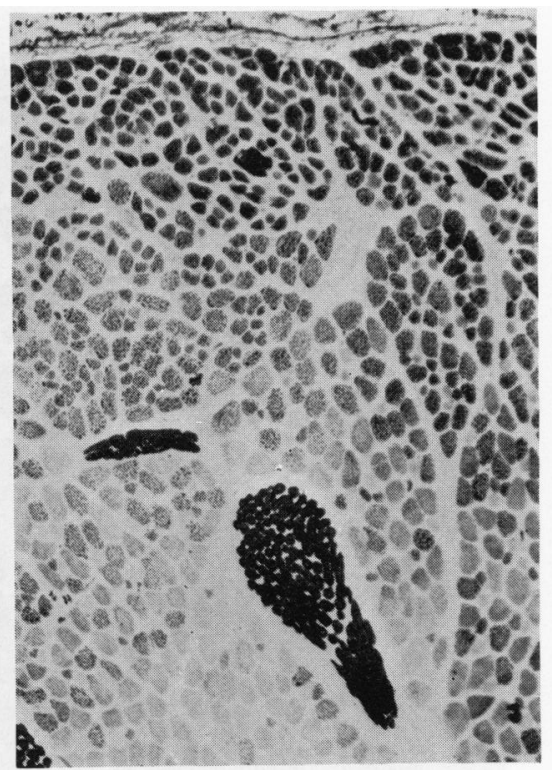

(b)

FIG. I 2 Sudan black $B . \quad \times 8$ o. Distribution of coarse fibres

(a) Control levator palpebrae superioris

(b) Control superior rectus 
The coarse fibres at the peripheries of the oblique and rectus muscles showed the highest glycolytic capacities, whereas these types stained uniformly for glycogen and phosphorylase in the levator muscles (Fig. 13).
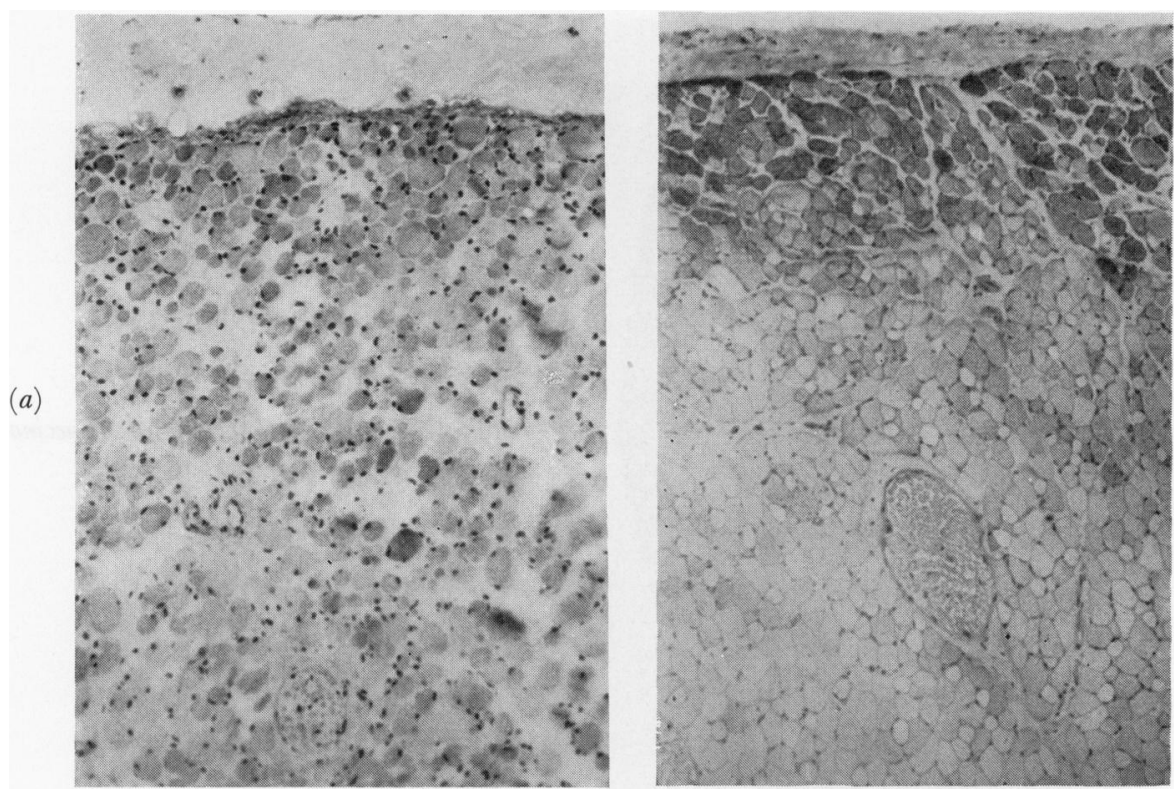

(b)

FI G. I 3 Periodic acid-Schiff. Haematoxylin counterstain. $\quad \times 80$. Distribution of glycogenrich fibres

(a) Control levator palpebrae superioris

(b) Control superior rectus

The arrangement of the fibres in three zones in the oblique and rectus muscles was also demonstrable with low-power views of the histochemical preparations. The peripheral and

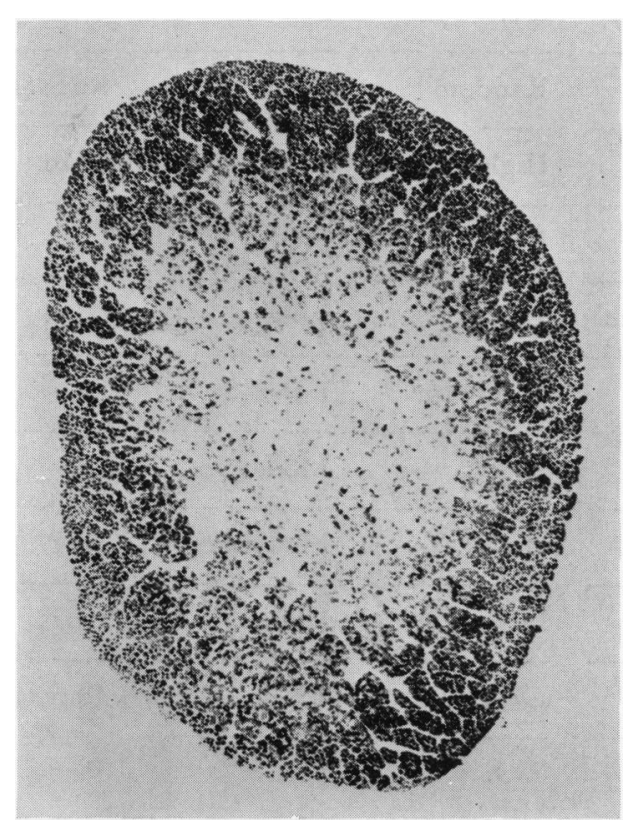
intermediate zones formed a double ring around the central region in transverse sections taken near the origins of the muscles (Fig. I4).

FIG. I 4 Control inferior rectus. Sudan black B. $\times$ Io. Section near muscle origin 
As the rectus and inferior oblique muscles were traced anteriorly, the ring configuration gave way to an arc and the central zone became directly applied to the globe (Fig. I5). In the superior oblique muscles, the ring appearance was seen in all transverse sections.

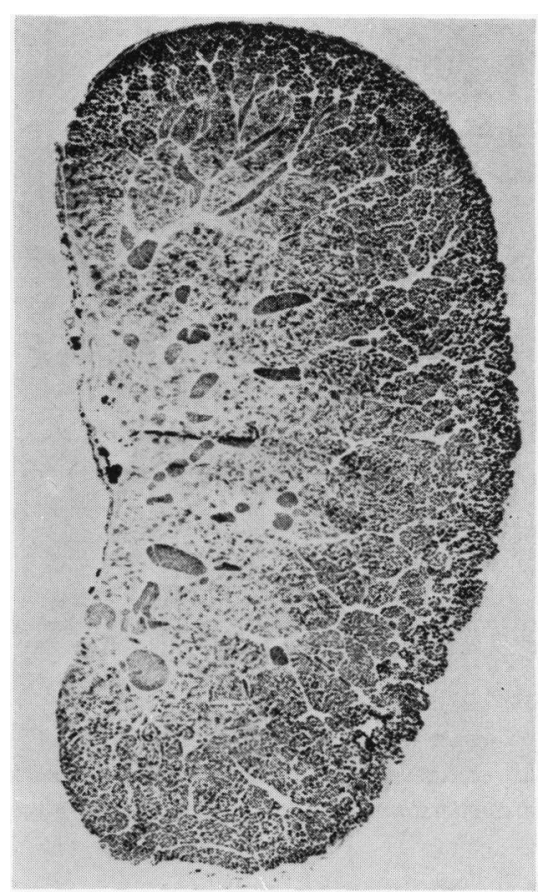

FIG. I 5 Control inferior rectus. Succinate dehydrogenase. $\times$ I 0. Mid-belly section

The histochemical profiles of the extraocular muscles are summarized in Tables I and II.

Table I Histochemical profiles of levator palpebrae superioris muscles

\begin{tabular}{|c|c|c|c|}
\hline Fibre type & Coarse & Fine & Granular \\
\hline Distribution & Random & Random & Random \\
\hline ATPase & High & Low & High \\
\hline ATPase with acid pre-incubation & Low & High & Low \\
\hline$\alpha$-glycerophosphate dehydrogenase & Low & Low & High \\
\hline Glycogen and phosphorylase & Moderate & Low & Low \\
\hline Oxidative enzymes & High & Moderate & Low \\
\hline Neutral lipids & High & Moderate & Low \\
\hline Esterases & Moderate & High & Low \\
\hline Nerve endings & En grappe & En plaque & En plaque \\
\hline Capillaries & Rich & Scanty & Scanty \\
\hline
\end{tabular}


Table II Histochemical profiles of oblique and rectus muscles

\begin{tabular}{|c|c|c|c|}
\hline Fibre type & Coarse & Fine & Granular \\
\hline Distribution & $\begin{array}{l}\text { Mostly peripheral and } \\
\text { intermediate zones }\end{array}$ & Random & Central zone \\
\hline ATPase & High & Low & High \\
\hline ATPase with acid pre-incubation & Low & High & Low \\
\hline$\alpha$-glycerophosphate dehydrogenase & Low & Low & High \\
\hline Glycogen and phosphorylase & $\begin{array}{l}\text { Ranging from high in } \\
\text { peripheral zone to low in } \\
\text { central zone }\end{array}$ & Low & Low \\
\hline Oxidative enzymes & High & Moderate & Low \\
\hline Neutral lipids & High & Low & Low \\
\hline Esterases & Moderate & High & Low \\
\hline Nerve endings & En grappe & En plaque & En plaque \\
\hline Capillaries & Rich & Scanty & Scanty \\
\hline
\end{tabular}

\section{(c) Muscle spindles}

No spindles were recognized in the extraocular muscles of the baboon. In the human material, they were found in the peripheral and intermediate zones in a plane near the muscle origins. They contained four to eight fibres and in some cases their capsules were split to include two or three extrafusal fibres (Fig. I6). The morphological properties of the intrafusal fibres were uniform and they could not be subdivided into nuclear bag or nuclear chain types. With the ATPase reaction, the spindle fibres showed a high intensity of staining which was reversed with acid pre-incubation (Fig. I6).

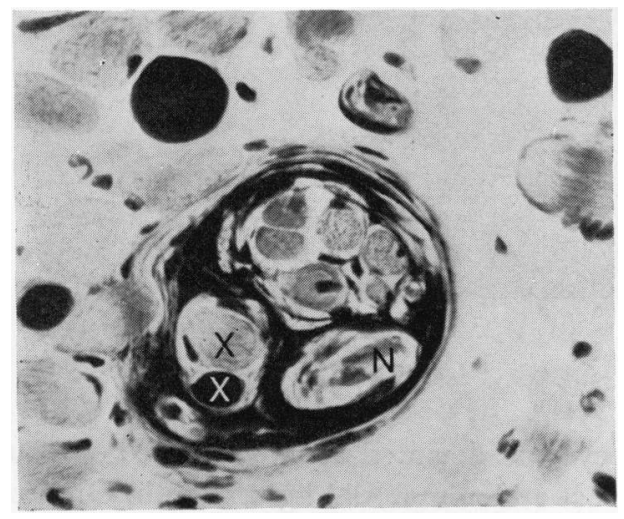

FIG. I 6 Human inferior oblique. Acid-activated ATPase (sequestrate, pH 4:35). Haematoxylin counterstain. $\times 340$. Spindle. All six intrafusal fibres stain lightly. Capsule split to include two extrafusal fibres $(X)$ $\mathrm{N}=$ nerve 
They reacted poorly for glycogen, phosphorylase, lipids, and $\alpha$-glycero-phosphate dehydrogenase, but showed intense reactions for oxidative enzymes and non-specific esterases (Fig. I7).

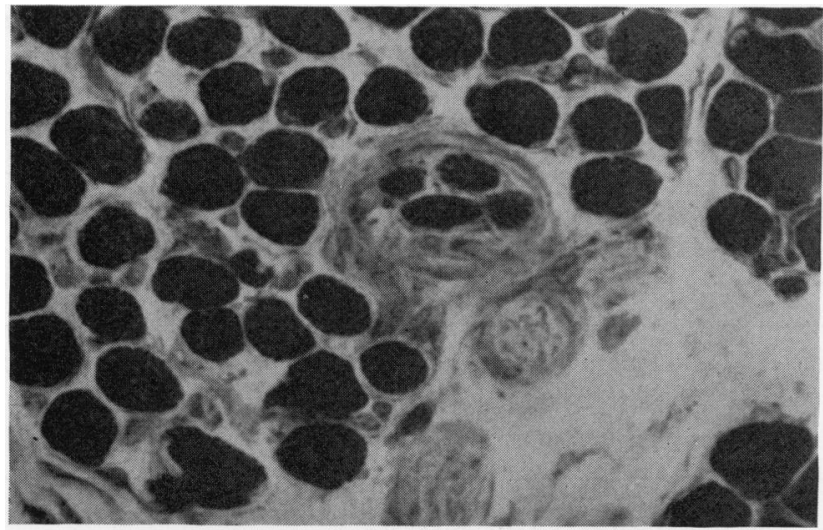

(a)

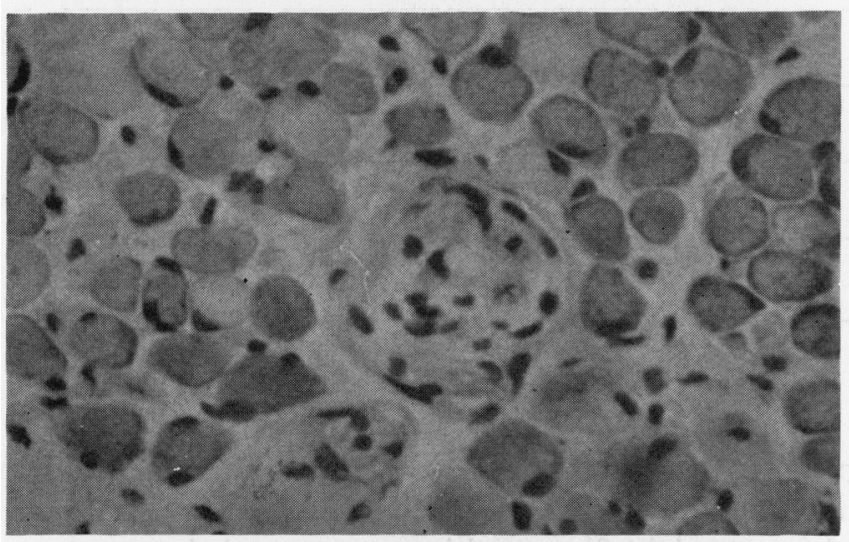

FIG. 7 Human inferior oblique. Identical spindle. $\times 240$ (a) $\mathrm{NADH}_{2}$ tetrazolium re-

(b) ductase

(b) Periodic acid-Schiff. Haematoxylin counterstain

(c) Phosphorylase

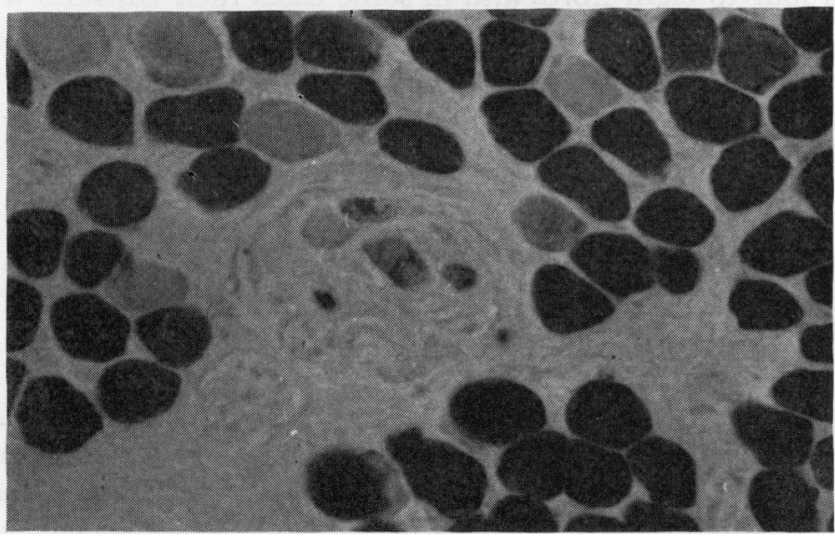

$(c)$

(2) EXPERIMENTAL ANIMALS

(a) Clinical observations (Figs I 8 to 22)

Immediately after operation, the right eye was deviated downwards and outwards and the pupil was dilated and unreactive to direct and consensual light stimuli. On recovery from 
FIG. I 8

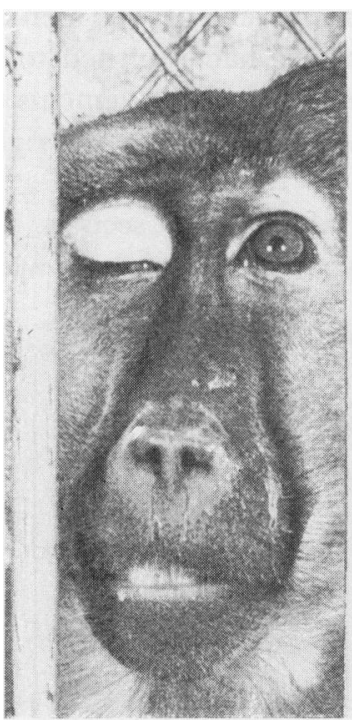

FIG. I9

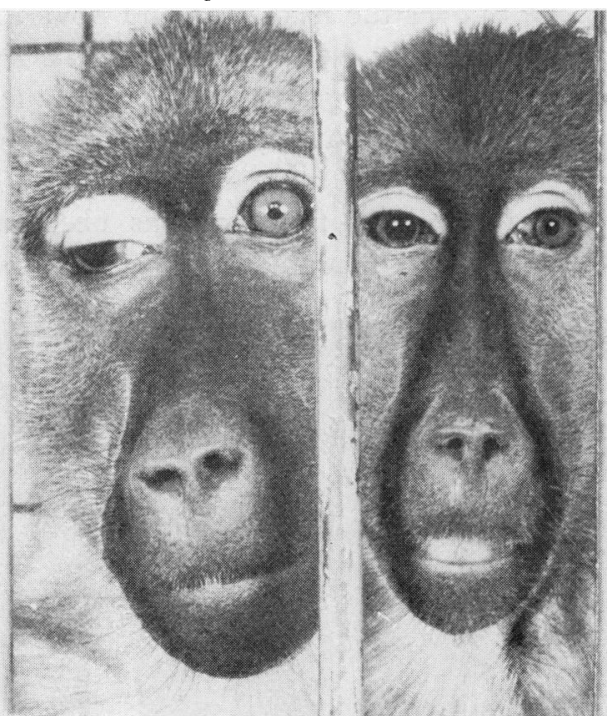

FI G. 2 I

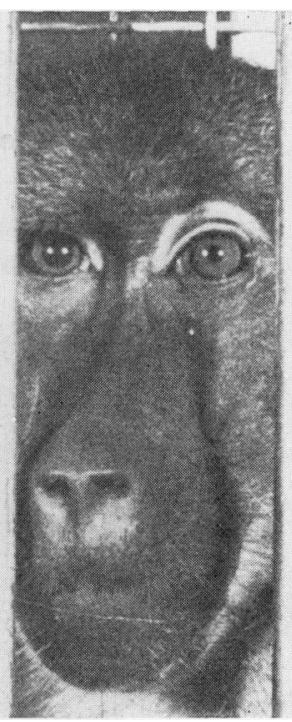

FIG. I 8 Baboon attempting to look upwards I day after right oculomotor nerve section

FIG. I 9 Baboon attempting to look ahead I month after right oculomotor nerve section

FIG. 20 Baboon attempting to look to the left 6 weeks after right oculomotor nerve section

FIG. 2 I Baboon looking to the left I year after right oculomotor nerve section

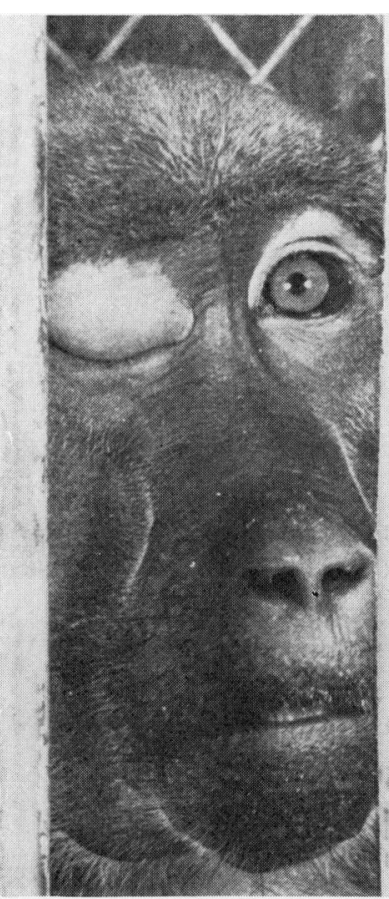

(a) I day

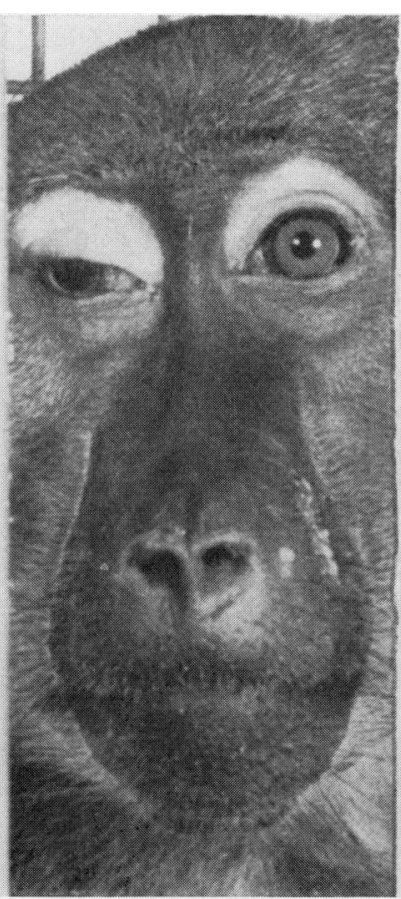

(b) I week

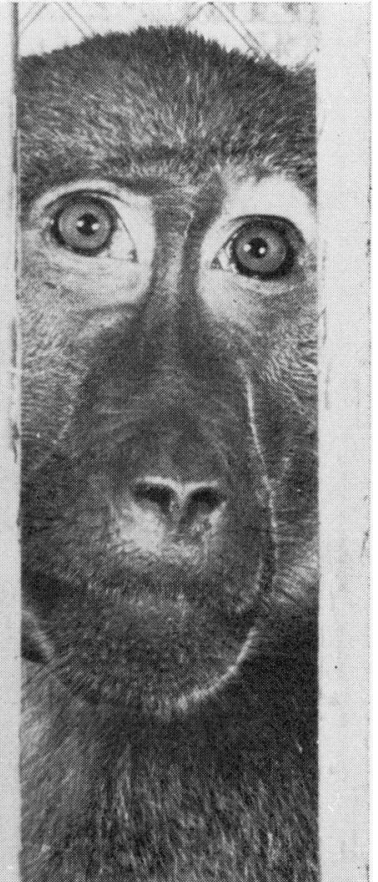

(c) I month after right oculomotor nerve crush

FIG. 22 Baboon attempting to look ahead 
anaesthesia, all the animals showed complete ptosis on the right side (Fig. I8). This improved gradually over the next year and obvious improvement was seen as soon as I month after operation (Fig. I9). By 6 weeks, the right eye had returned to the primary position (Fig. 20). There was gradual recovery of the range of eye movements over the rest of the 2-year period of observation, although they remained slower on the right side (Fig. 21 ).

No recovery of pupillary function was observed after nerve section. The clinical findings immediately after oculomotor nerve crush were identical to those which followed

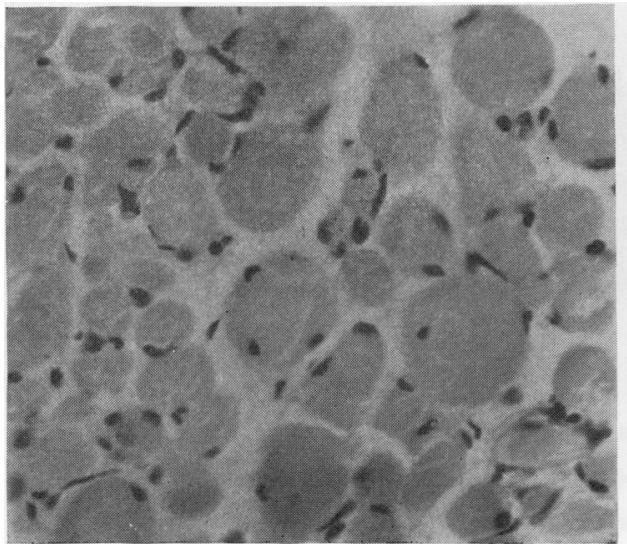

(a) Control

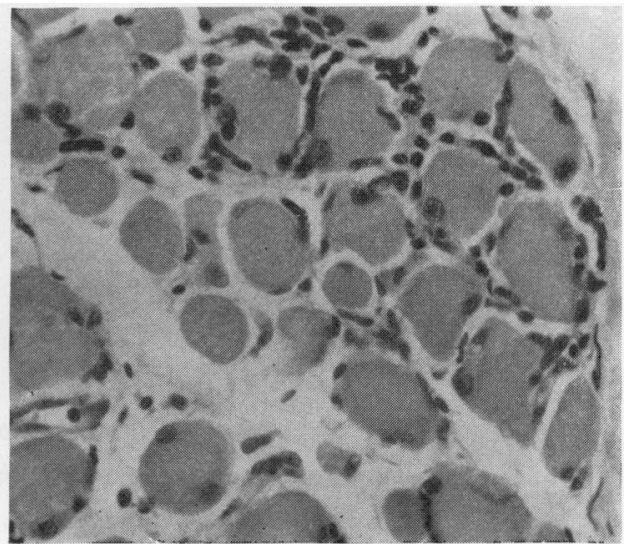

(b) 3 weeks after nerve section

FIG. 23 Medial rectus. Paraffin section. Haematoxylin and eosin. $\quad \times 280$

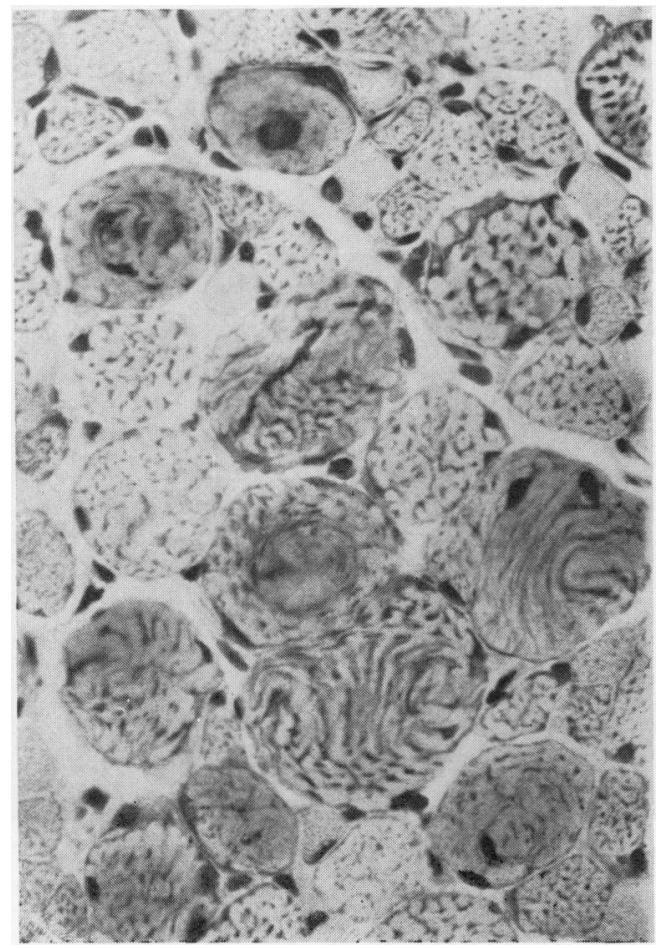

FIG. 24 Inferior rectus. Haematoxylin and eosin. $\times 340.3$ weeks after nerve section 


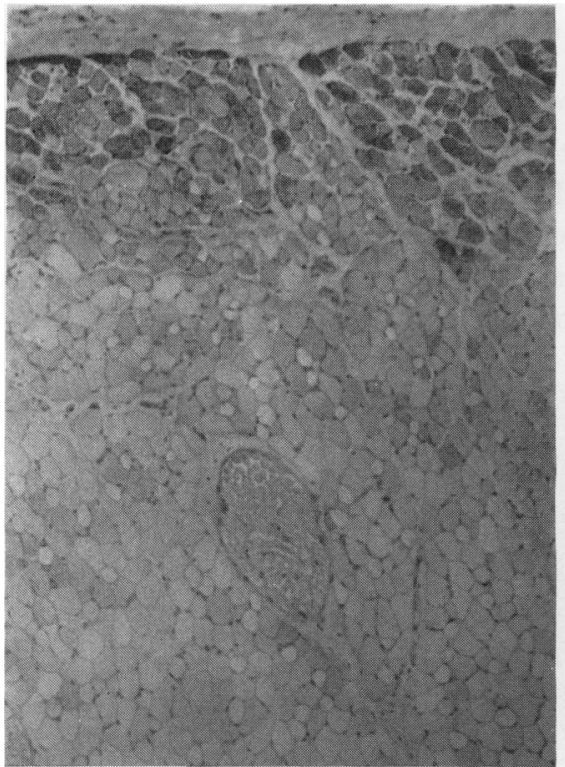

(a) Control

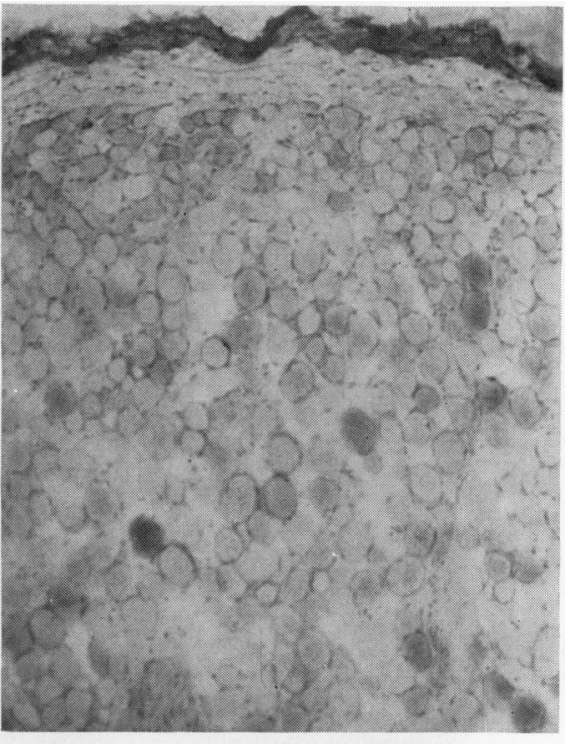

(b) 3 months after nerve section

FI G. 25 Superior rectus. Periodic acid-Schiff. Haematoxylin counterstain. $\quad \times 80$

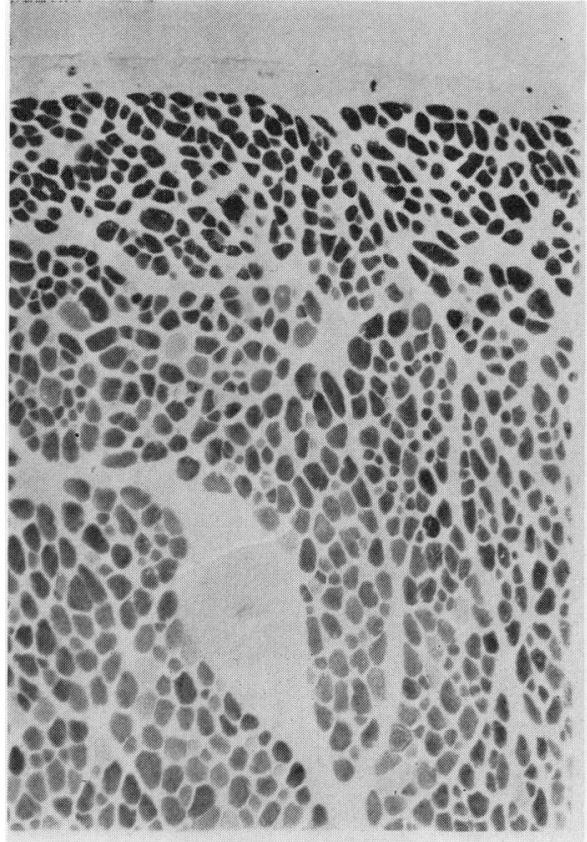

(a) Control

rig. 26 Superior rectus. Phosphorylase. $\times 80$

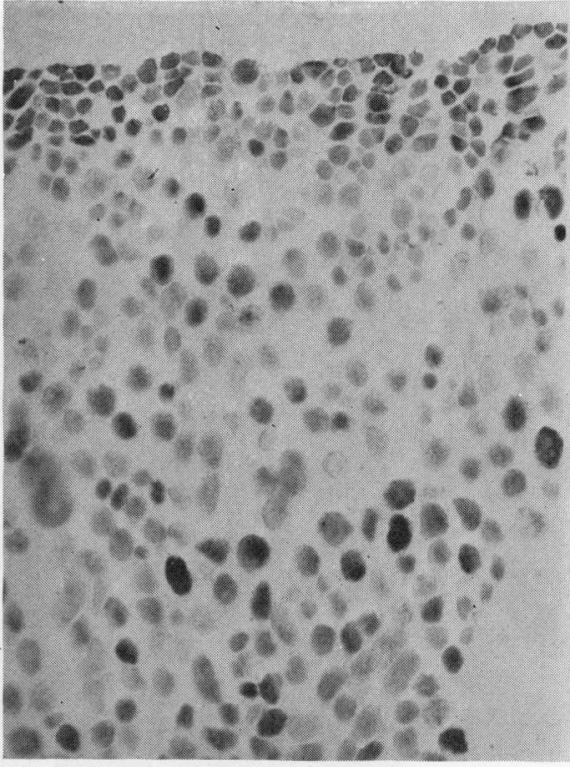

(b) 3 months after nerve section 
nerve section. However, there was gradual and complete recovery of the ptosis, ocular movements, and pupillary function over a period of I month (Fig. 22).

(b) Morphological and histochemical observations

In the two animals killed 3 weeks after nerve section, no wasting was detected on dissection of the muscles of the right orbit and the cut ends of the right oculomotor nerve were still separated. The muscles of the right orbit, other than the lateral rectus and superior oblique, showed uniform microscopic changes. The fibre nuclei were swollen and their nucleoli prominent. There was marked infiltration of round cells between the fibres (Fig. 23).

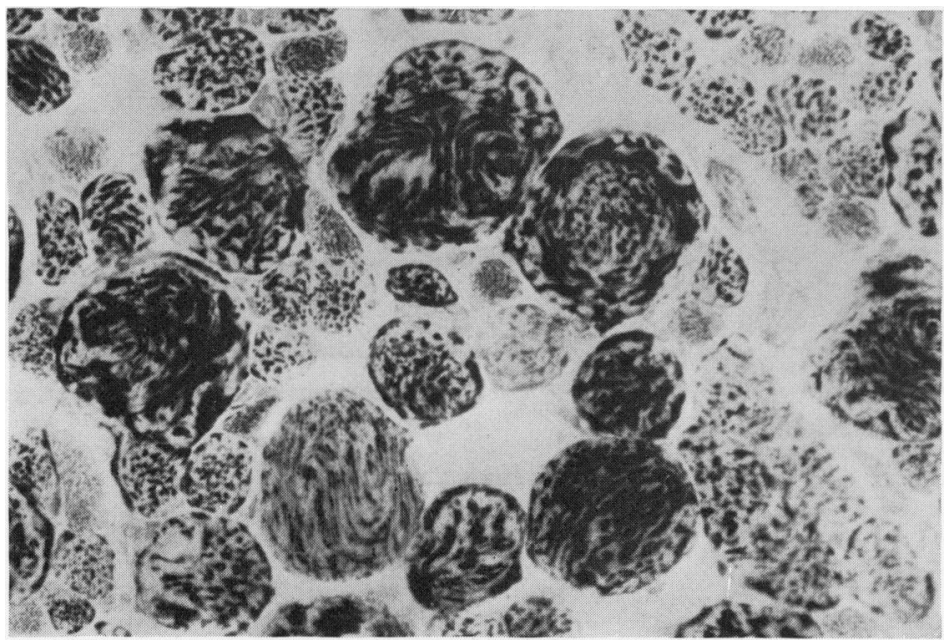

FIG. 27 Inferior rectus. $\mathrm{NADH}_{2}$ tetrazolium reductase. $\times 340.3$ weeks after nerve section

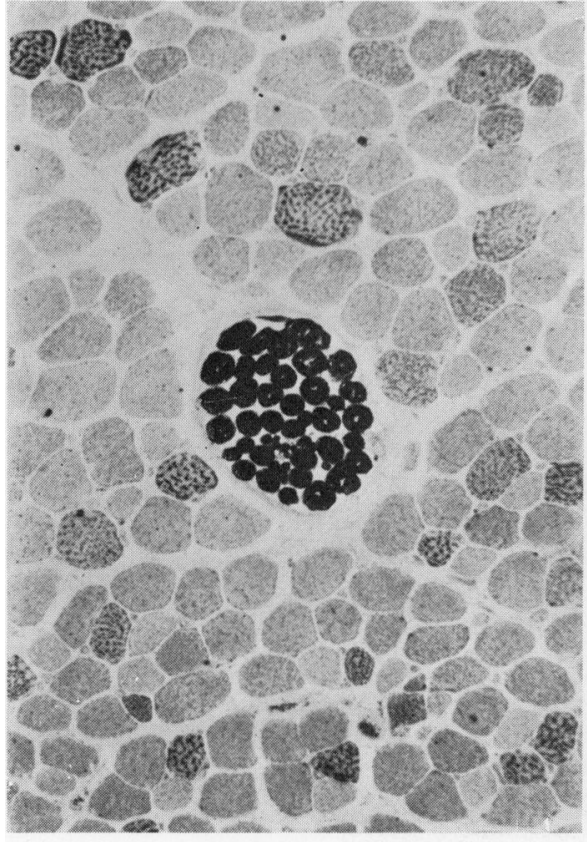

(a) Control

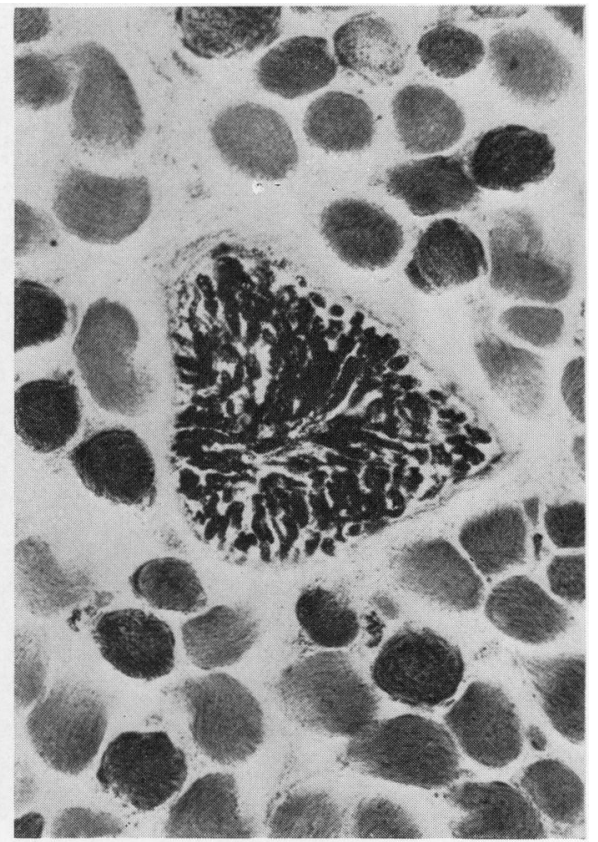

(b) 3 weeks after nerve section

FIG. 28 Superior rectus. Sudan black $B . \quad \times 340$ 
The architecture of the reticulum of the coarse fibres was disrupted and appeared whorled in transverse section (Fig. 24). The fine and the ganular fibres showed a coarsening of their reticulum. The intramuscular nerves showed loss of myelin.

In the denervated muscles, there was loss of glycogen and phosphorylase, particularly from those fibres which normally stain intensely (Figs 25 and 26).

The oxidative, Sudan black, and $\alpha$-glycerophosphate dehydrogenase preparations confirmed the disruption of the reticulum (Fig. 27).

The Sudan stain also showed myelin debris in the intramuscular nerves indicating Wallerian degeneration (Fig. 28). No change was seen in the esterase staining or in the capillary networks. There was no apparent alteration in the sizes or distribution of the fibre types. No increase in connective tissue was found.

In the two animals killed at 3 months, the muscles showed no change in bulk, but the cut ends of the right oculomotor nerve had become reconnected (Fig. 29). The morphologi-

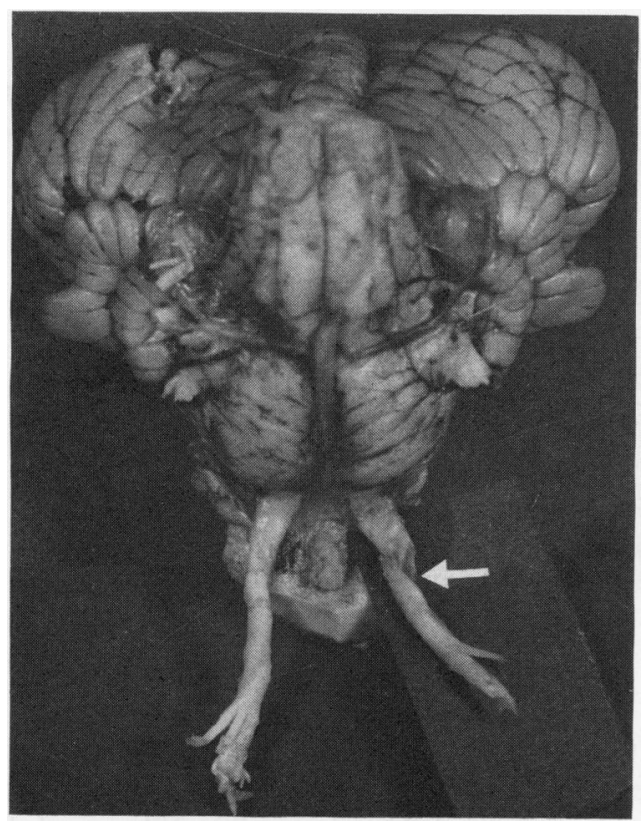

F I G. 29 Dissection showing reconnected cut ends of right oculomotor nerve (arrowed) 3 months after operation

cal and histochemical properties of the denervated muscles were similar to those found at 3 weeks, with the exception that the intramuscular nerve fibres had remyelinated.

The findings in the muscles examined at 6 months, I year, and 2 years were comparable one to another. The cut ends of the right oculomotor nerves were reconnected and the intramuscular nerves showed no abnormality. No nuclear changes or cellular infiltration were seen and the fibres showed no architectural abnormalities. The striking change in the muscles was the appearance of groups of up to thirty fine fibres with a wide range of diameters (Fig. 3o, overleaf). No grouping of the coarse or the granular fibres was detected and no selective type atrophy was found.

In the muscles of the two animals examined 3 months and 9 months after nerve crush, no changes in morphological or histochemical properties were encountered. In particular, the type distributions and the intramuscular nerves appeared to be normal. In all experimental animals, the right lateral rectus and superior oblique muscles showed no abnormality. 

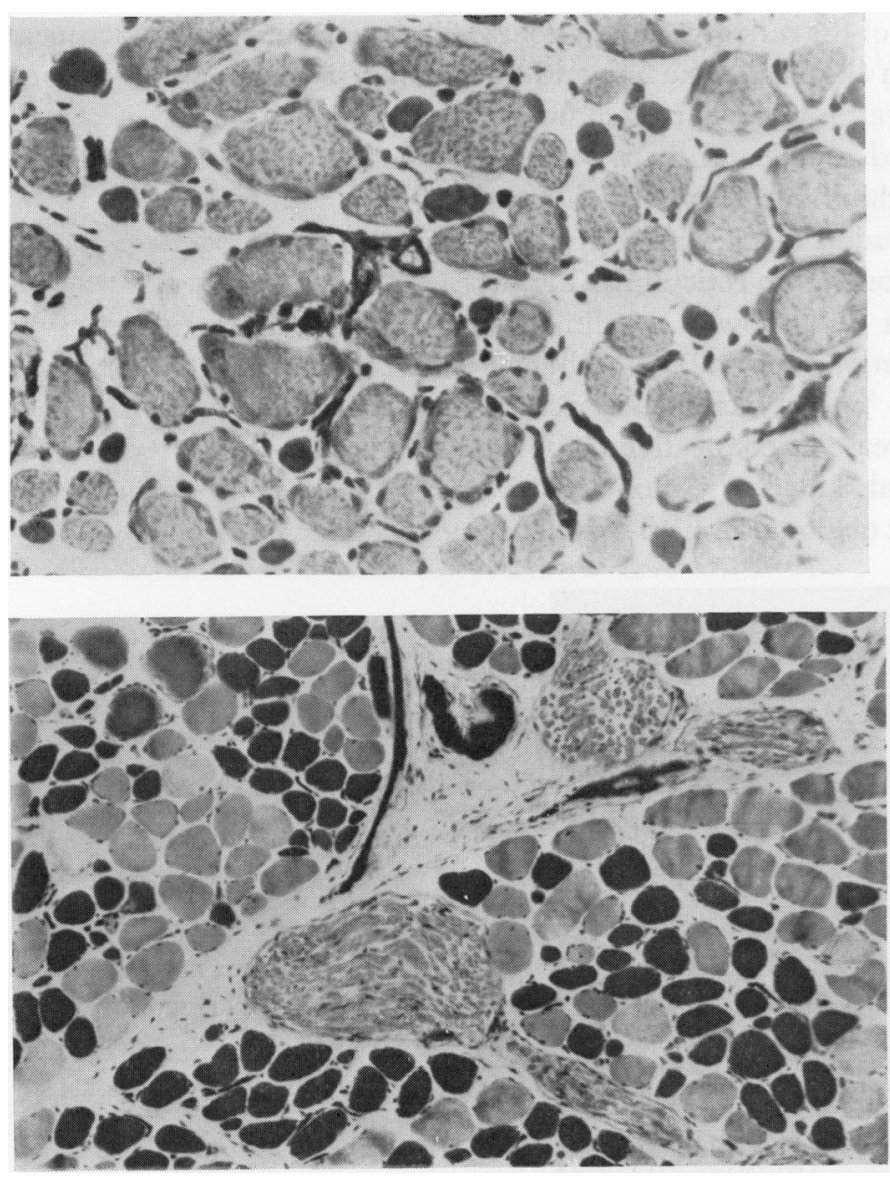

(a)

FI G. 30 Superior rectus. Acidactivated ATPase (sequestrate, $\mathrm{pH}_{4} \cdot 35$ ). Haematoxylin counterstain. $\times 240$

(a) Control

(b) Grouping of fine fibres 2 years after nerve section

\section{Conclusions}

Many previous morphological and ultrastructural studies have described a dual type of Felderstruktur and Fibrillenstruktur fibres in the extraocular muscles of many species including man (Kruger, I929; Dietert, I965; Brandt and Leeson, I966; Pilar and Hess, I966; Namba, Nakamura , and Grob, I968). Later reports have described a triple type system in which the Fibrillenstruktur fibres were subdivided (Cheng and Breinin, I966; Mayr, Stockinger, and Zenker, I966; Cheng-Minoda, Ozawa, and Breinin, I968). Three fibre types were found in the present study which correspond to the results of the latter workers. Histochemical studies of extraocular muscles have been few. Yellin (1969) showed three fibre types in the rat using the ATPase reaction. Miller (1967) claimed to have found five types in the Rhesus monkey according to histochemical and ultrastructural differences but did not use the ATPase reaction. The three-type system does not preclude further subdivision according to more subtle differences, such as those described by Miller ( 1967 ), which probably represent minor variations according to the location of the Fibrillenstruktur fibres within the muscles.

The physiological typing of the extraocular muscle fibres has been the subject of controversy and comparisons with the skeletal muscles of the limbs have frequently been made. The presence of multiple endings en grappe in the coarse (Felderstruktur) fibres and their slow 
graded response to the local application of acetyl choline has led to their being compared with the slow-tonic fibres found in the iliofibularis muscle of certain amphibia (Duke-Elder and Duke-Elder, I930; Kern, I965). Slow-tonic fibres are incapable of propagating action potentials and respond to repeated neural stimulation with a slow contracture (Kuffler and Vaughan Williams, I953). In mammalian limb muscles, the motor units are all of the 'twitch' type and possess single endings en plaque. They are capable of propagating action potentials and respond to stimulation with a single contraction in an all-or-none fashion (Close, 1967). In primates, two main divisions are recognized and are termed 'fast-twitch' and 'slow-twitch' according to differences in their contraction speeds, thresholds for stimulation, and resting potentials (Eberstein and Goodgold, I968). The Fibrillenstruktur fibres, which in the present study have been subdivided into fine and granular types, were assumed to have a 'fast-twitch' mode of action as their endings en plaque and sarcoplasmic reticulum resembled those of the fast-twitch fibres of the limbs.

A simple division into slow-tonic and fast-twitch systems was supported by the electrophysiological studies of Hess and Pilar (1963) in the cat, and seemed to correspond to the dual-type system of earlier morphological reports. However, Bach-y-Rita and Ito (I966), while agreeing about the properties of the fast-twitch units, considered the slow units to be of the twitch type also. An attempt to reconcile the two views was made by Peachey (ig68), who suggested that the differences in the electrophysiological results were due to different fibres being sampled and to differences in the sizes and location of the fibres within the muscles studied. The three types of motor unit were thus indirectly correlated with the three morphological types of fibre.

The histochemical properties of limb muscles, and to some extent their physiological characteristics, are influenced by their motor nerve supply. This has been shown by crossinnervation studies in the cat and rat (Dubowitz, 1967; Romanul and van der Meulen, 1967). Most early attempts at correlating the histochemical properties of muscle fibres to the physiological role of the motor units to which they belong have come about indirectly by extrapolation of data obtained from studies on whole muscles and their nerves. Later studies of single motor units have led to more direct correlations. It was shown that the muscle fibres belonging to a particular motor unit were of uniform histochemical type (Brandstater and Lambert, I968; Kugelberg and Edstróm, I968). In a study of single motor units in cat gastrocnemius muscles, further correlations were made between the motor unit physiology and the histochemistry of the muscle fibres (Burke, Levine, Zajac, Tsairis, and Engel, I97I). In this species, the histochemical properties of the muscles resemble those of primates (Brooke and Kaiser, I970; Durston, I97 I). Three populations of motor unit were found. The muscle fibres of the slow-twitch units stained poorly for ATPase activity (histochemical type I). They showed a high intensity for oxidative enzymes and were relatively resistant to fatigue. The fast-twitch fibres all showed a strong reaction for ATPase (histochemical type II) but could be subdivided. Those fast-twitch fibres with high oxidative enzyme activity were relatively resistant to fatigue during repetitive nerve stimulation (type IIB), whereas those which stained poorly (type IIA) belonged to units which fatigued more rapidly. Against this background it is possible to discuss the physiological role of the extraocular fibre types, even though their histochemical characteristics differ significantly from those of limb muscles.

The coarse fibres make up the bulk of the extraocular fibre population and were in a particularly high proportion in the lateral rectus and levator palpebrae muscles. Their multiple endings en grappe suggest that they are capable of graded contractions similar to those demonstrated pharmacologically in the cat, dog, and rabbit (Duke-Elder and Duke- 
Elder, I930; Kern, I965). Although they resemble slow-tonic fibres morphologically, their histochemical properties are quite different, for in the frog and toad the slow-tonic fibres show poor reactions for ATPase, lipids, oxidative enzymes, glycogen, and phosphorylase (Lännergren and Smith, I966; Engel and Irwin, 1967). The intense ATPase activity at $\mathrm{pH} 9.4$ shown by the coarse fibres indicates that they may have a relatively fast speed of contraction in certain conditions (Bárány, I967). Their high oxidative capacities and rich capillary networks suggest that they use the Krebs cycle as an energy source, are capable of incurring an oxygen debt, and thus are relatively resistant to fatigue (Romanul, I964; Romanul, I965). They are probably capable also of utilizing lipids as a source of energy. Their staining intensities for glycogen and phosphorylase vary with the location of the fibre. They show most reaction in the levator muscles and at the periphery of the oblique and rectus muscles. The coarse fibres in these positions are thus probably capable of anaerobic activity and use the Embden-Meyerhof pathway as well as the Krebs cycle (Romanul, 1964).

The fine fibres share several histochemical properties with the slow-twitch type I fibres of primate limb muscles. They stain poorly for $\alpha$-glycerophosphate dehydrogenase, glycogen, phosphorylase, and ATPase at $\mathrm{pH} 9.4$. They react strongly for acid-activated ATPase and non-specific esterases. They differ, however, in staining poorly for lipids. The fine fibres would seem to be of relatively small importance in extraocular muscle function. They are few in number and were absent altogether from two of the baboon levator muscles. In common with the fast-twitch type II fibres of primate limb muscles, the extraocular granular fibres stain intensely for ATPase and $\alpha$-glycerophosphate dehydrogenase. They differ in showing poor reactions for glycogen and phosphorylase. Their main source of energy is probably oxidative.

It has been held that the various observable eye movements were each the function of one type of motor unit and thus of a particular fibre type (e.g. Jampel, 1967). Recently, however, this view has been challenged. It has been suggested from work in the monkey that all abducent and oculomotor units participate in every kind of eye movement, whether it be fixation, pursuit, saccadic, vergent, or vestibular, and so each of these is not the exclusive property of one fibre type (Fuchs and Luschei, I970; Robinson, 1970; Schiller, 1970; Robinson and Keller, 1972). If this is so, what are the roles of the fibre types in a composite muscle? Experiments on the limb muscles of the rat have suggested that during a contraction, the low threshold units to which the slow-twitch type I fibres belong are the first to be recruited. As the contraction increases, the fast-twitch type II fibres are brought into action (after Edström and Kugelberg, 1968). It is probable that a similar process operates in the extraocular muscles. Two population peaks have been demonstrated in the abducent and oculomotor units of monkeys according to their thresholds for stimulation (Fuchs and Luschei, I970; Robinson, 1970; Schiller, 1970; Robinson and Keller, 1972). The 'low-threshold' fibres are likely to be those which are relatively resistant to fatigue. The coarse fibres appear to possess the biochemical properties for this. Such activity would be seen in fixation movements which are constantly active during waking hours. The anaerobic metabolism of the coarse fibres located at the periphery of the obliques and recti suggests that they contract late and are 'protected' when the blood supply of the muscle is mechanically reduced. The granular fibres are likely to act in a fast-twitch manner and to be brought into action mostly during brief eccentric movements or saccades in the 'on' direction. The point at which the fine fibres are recruited is difficult to infer from their histochemical properties. It is possible that they are vestigial or have an afferent role.

Spindles have been found in the extraocular muscles of sheep, goats and many higher 
primates including man (Cooper, Daniel, and Whitteridge, I955). In man, ocular intrafusal fibres are less differentiated than those of the skeletal muscles in that they cannot be divided into nuclear bag and nuclear chain types but fall into an intermediate form (Cooper and Daniel, 1949). Their histochemical properties are also uniform. The functions of the extraocular spindles are not clear. The orbital muscles act upon a constant load and possess no stretch reflex (Robinson and Keller, 1972). They may be concerned with monitoring contraction speeds or with feed-back projections to the cerebellum (Fuchs and Kornhuber, I969). Although no spindles are present in the extraocular muscles of the cat and the baboon, this does not preclude an afferent role for some of the fibres, but the fact that they are not encapsulated makes them difficult to identify. Afferent function is also subserved by the spiral endings related to the central fibres.

The partial recovery of ocular movements and levator function by 6 weeks after nerve section indicates that re-innervation had occurred during this interval, and this was confirmed by the findings on dissection and microscopy at 3 months. The slower speed of movements observed in the right eye at 2 years may be related to a change in the type ratios. Although total counts were not made, it was obvious that the number of fine fibres had increased as a result of re-innervation. The persistence of a dilated, non-reactive pupil for 2 years after nerve section indicates that the parasympathetic nerve fibres had not recovered to the same extent as the oculomotor units, if at all. The complete recovery of oculomotor and parasympathetic function in I month and the absence of type grouping after a simple crush lesion suggest that all the fibres contained in the oculomotor nerve possess considerable powers of regeneration provided the continuity of their sheaths is not interrupted.

At 3 weeks and 3 months after nerve section, the morphological changes resembled those seen in baboon limb muscles denervated for the same period (Durston, I97 I). These early findings were also similar to those described in the extraocular muscles of the dog after experimental denervation (Drachman, Wetzel, Wasserman, and Naito, I969). Plates of atrophic fibres, considered a sign of denervation in limb muscles, are not seen in the muscles of the orbit. This may in part be due to difficulty in recognizing such changes in muscles which normally contain fibres of widely ranging diameters, but in the present experiments and those of Drachman and others (I969) early re-innervation was not prevented. Although there was early loss of glycogen and phosphorylase from those fibres which normally stain intensely, few conclusions could be drawn concerning the trophic role of the oculomotor nerve as the muscles were not completely denervated for a sufficiently long period and no atrophy was observed. The type grouping seen 6 months, I year, and 2 years after nerve section affected the fine fibres to the greatest extent. This suggests that the motor axons which normally supply fine fibres regenerate more quickly or sprout more readily and re-innervate coarse and granular fibres to impose upon them the histochemical characteristics normally associated with the fine type. A similar phenomenon has been described in limb muscles as a result of experimental reinnervation (Karpati and Engel, I968). The finding compares with the more extensive type I grouping seen in limb muscles after partial denervation when the lesion responsible affects the peripheral nerve rather than more central pathways (Morris and Raybould, I97I). This then is further evidence for considering the fine fibres to be homologous with the type I.

\section{Summary}

Three types of fibre have been described in the extraocular muscles of the baboon and man 
using a variety of morphological and histochemical techniques. Their histochemical profiles have been discussed in relation to their physiology. It has been suggested that their differences are related to the times of recruitment of the motor units to which they belong. The effects of oculomotor nerve section and crush have been described and compared with the experimental results of other workers.

I am grateful to Prof. R. W. Gilliatt and Dr. J. A. Morgan-Hughes for their interest and encouragement. I thank Mr. Lindsay Symon for performing the initial operations on the baboons and Mr. Michael Sanders for providing the human material. The histochemical procedures were undertaken in a laboratory equipped by the Muscular Dystrophy Group of Great Britain and a personal research grant from the National Fund for Research into Crippling Diseases is gratefully acknowledged.

Technical assistance was provided by Miss Carole Aldridge, Miss Jennifer Andrews, and Miss Sally Sinclair. The report forms part of a dissertation for the degree of M.D. of the University of Cambridge.

\section{Discussion}

STRACHAN Was there any increase in the connective tissue in the denervated muscle?

DURSTON None was seen in my material, but if I had kept the muscles denervated for a longer period of time, then an increase in connective tissue might have been seen.

ABRAHAms Do all the fibres in the extraocular muscles run from end to end of the muscle?

DURSTON In transverse sections taken very near the origins and insertions of the muscles there was evidence that some fibres were shorter than others and that they were inserted into the globe at different points.

BRON Was activity of the animals controlled in these studies before they were killed? This might have altered the levels of substrates such as glycogen within the muscles, and so given a false impression of enzyme activity.

DURSTON There is no direct evidence of this, but all the findings were consistent in these animals. There is no doubt that the glycogen content is the least satisfactory of histochemical stains for typing fibres, as it has been shown in limb muscles that the staining intensities are veryn variable, and could easily be altered by nerve stimulation or section. It was possible that stimulation of the nerve could occur as the muscle was being removed.

HUBER How are the electrophysiological changes in myopathies related to the histochemical changes in the muscles?

DURSTON I have no information about the electromyograms of ocular muscles in myopathies, but the morphological response which is produced by nerve section has long been thought of as myopathic. The fact that nuclear changes and cellular infiltration could be produced by experimental denervation, the occurrence of peripheral neuropathic features with progressive ophthalmoplegia, and the findings in some patients reviewed by Kiloh and Nevin (195I) of degenerative changes in the oculomotor nuclei, all suggest that the syndrome of progressive ophthalmoplegia has more than one aetiology. It is possible that some are truly dystrophic and others neuropathic. 